Mitteilungen der Österreichischen Geographischen Gesellschaft,

155. Jg. (Jahresband), Wien 2013, S. 61-90

\title{
Das regionale Dilemma der Europäischen Union: RÄUMLICHE UNGLEICHGEWICHTE IN DER GEGENWÄRTIGEN WIRTSCHAFTSKRISE
}

\author{
Robert MusiL, Wien* \\ mit 6 Abb. und 4 Tab. im Text
}

\section{INHALT}

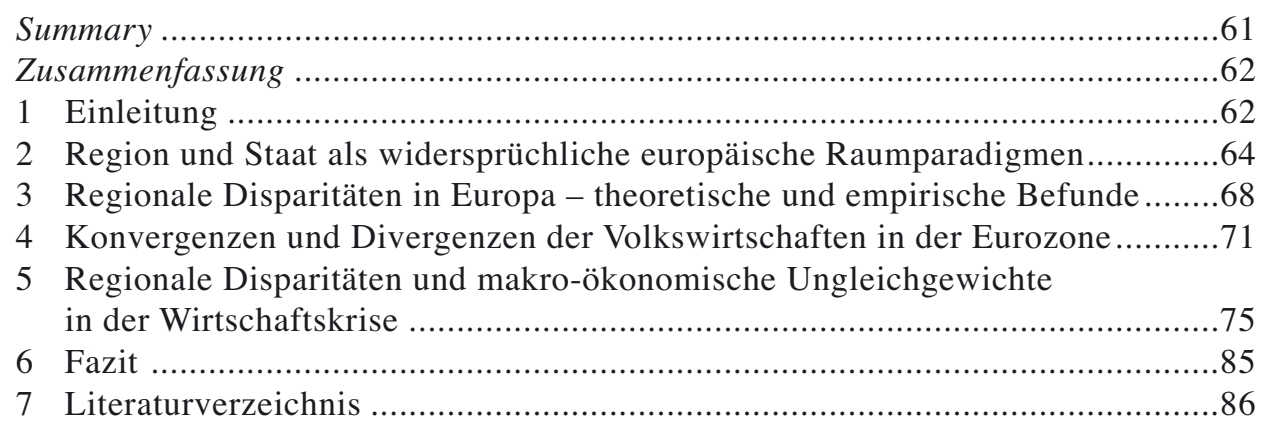

\section{Summary}

The regional dilemma of the European Union: Spatial disparities in the recent economic crisis

The recent economic crisis has led to a growing macro-economic imbalance among the countries of the euro zone. Against this background, this paper discusses the impact of macro-economic developments on regional disparities, focusing on the periods before and since the recent crisis. After discussing the region as a symbol of the European integration process and as a paradigm in social sciences, the empirical section of this paper analyses the correlation between national and regional disparities. However, spatial developments are not examined on the level of the European Union, but rather in the respective national context.

\footnotetext{
* Mag. Dr. Robert MusiL, Institut für Stadt- und Regionalforschung, Österreichische Akademie der Wissenschaften, Postgasse 7/4/2, A-1010 Wien; E-Mail: robert.musil@oeaw.ac.at, http://www.oeaw.ac.at/ isr
} 
The empirical findings demonstrate, firstly, that the recent crisis has led to an increasingly divergent spatial development within the twelve euro countries; and, secondly, that macro-economic trends on the national scale provide a "corridor" for regional and urban development processes to occur. The findings suggest that spatial developments should not be viewed only through the "regional lens", but that also macro-economic developments have to be considered.

\section{Zusammenfassung}

Die Eurozone ist seit 2007 von einer Wirtschaftskrise erfasst, die sich in einem zunehmenden makro-ökonomischen Ungleichgewicht zwischen den einzelnen Volkswirtschaften ausdrückt. Vor diesem Hintergrund diskutiert der Beitrag die Frage nach dem Einfluss der volkswirtschaftlichen Entwicklung auf die regionalen Disparitäten, wobei besonderer Fokus auf die Vorkrisen- und Krisenperiode gelegt wird. Nach einer Diskussion der Region sowohl als Symbol der europäischen Integrationspolitik als auch als Paradigma der räumlichen Sozialwissenschaften untersucht der Beitrag den Zusammenhang zwischen nationalen und regionalen Disparitäten. Räumliche Entwicklungen werden allerdings nicht auf der Ebene der Europäischen Union, sondern im jeweiligen staatlichen Kontext untersucht.

Die Analysen zeigen, dass die Krise tendenziell zu einer Divergenz der räumlichen Entwicklung in den zwölf untersuchten Euro-Staaten geführt hat, sowie, dass die makro-ökonomischen Trends einen „Korridor"vorgeben, in dem regionale und urbane Entwicklungsprozesse stattfinden. Die Ergebnisse legen es nahe, räumliche Prozesse nicht nur ausschließlich durch die „regionale Brille“ zu sehen, sondern die Entwicklungen der Volkswirtschaften sowie Rahmenbedingungen der nationalstaatlichen Ebene mitzudenken.

\section{Einleitung}

Mit der Einführung des Euro im Jahr 2002 hat der europäische Integrationsprozess seinen bisherigen Höhepunkt erreicht - demnächst (1. Jänner 2014) werden mit Lettland 18 EU-Mitgliedsländer die Gemeinschaftswährung eingeführt haben. Die gemeinsame Währung hat zwar zu einer Erleichterung des EU-Binnenhandels geführt, da Wechselkursrisiken und Währungstransaktionskosten entfallen sind (BECKER 2008, 7f.). Mit der Einführung des Euro haben sich jedoch auch die makro-ökonomischen Ungleichgewichte zwischen den Volkswirtschaften verstärkt. Auf der einen Seite weisen die ehemaligen Hartwährungsländer Leistungsbilanzüberschüsse und eine moderat wachsende oder schrumpfende Staatsverschuldung bei zugleich günstigen Finanzierungskonditionen an den Kapitalmärkten auf, während die Weichwährungsländer unter einer Exportschwäche, horrenden Arbeitslosenzahlen und ausufernden Staatsdefiziten leiden. 
Die Deutungen der Krise des Euroraumes sind konträr: Sie erfolgen entweder aus einer keynesianistischen oder einer monetaristisch-stabilitätsorientierten Perspektive. Die erste Lesart betont aufoktroyierte Spardiktate und die ungleiche Produktivitätsentwicklung, die durch die moderate Lohnpolitik in Deutschland verschärft wurde. Beides dient aus dieser Perspektive vor allem den partikularen Interessen der Hegemonie der Eliten der Hartwährungsländer (HADJimichalis 2011, S. 255). Gemäß zweiter, nämlich monetaristischer Lesart wird die Umsetzung von Strukturmaßnahmen, Budgetdisziplin sowie Sparprogrammen der öffentlichen Haushalte der PIIGS ${ }^{1)}$-Krisenstaaten gefordert (SINN 2012) - nicht zuletzt aufgrund der Verbindlichkeiten, die zu Lasten der Steuerzahler der Hartwährungsländer gehen.

Die aus letztgenannter Sichtweise abgeleiteten, stabilitätsorientierten Maßnahmen der EU-Troika scheinen jedoch die Rezession und deren soziale Folgen in den krisengebeutelten Volkswirtschaften des Südens zu verschärfen. Milliardenschwere Rettungspakete, die von den Steuerzahlern des Nordens um den Preis einer harten Austeritätspolitik finanziert werden, führen in den Ländern des Südens zu schwerwiegenden sozialen Verwerfungen und zur Destabilisierung des Sozial- und Gesundheitsbereiches (KaraniKolos et al. 2013, S. 2f.); der Begriff des „sozialen Krieges“ (Wolf 2009) scheint die Lage realistisch wiederzugeben. Die Verwerfungen entladen sich in Demonstrationen, Streiks sowie in geschürten Ressentiments gegen die Eliten des Nordens. Das Spardiktat „des Nordens“ auf der einen Seite und Reparationsforderungen für den Zweiten Weltkrieg durch griechische Politiker auf der anderen Seite (Aswestopoulos 2013) sind Ausdruck der Fragilität des Solidaritätsgedankens sowie des europäischen Friedensprojektes. Welche der beiden Perspektiven auch immer eingenommen wird - die Krise zeigt das Dilemma einer heterogenen Staatenunion von politisch eigenständigen, aber währungspolitisch verbundenen Volkswirtschaften.

Vor dem Hintergrund der sich in der Krise vertiefenden Spaltung zwischen Nord und Süd kommt der Frage nach der regionalen Kohäsion eine neue Relevanz zu, die über das Auf und Ab regionaler Disparitäten im Verhältnis zum EU-Durchschnitt hinausgeht (vgl. diverse Kohäsionsberichte, etwa 5. Kohäsionsbericht, EuropäIsche Kommission 2010). Die „regionale Brille“, durch die Regionalökonomen und Geographen die Europäische Union lange und intensiv studiert haben, ist aufgrund der massiven Verwerfungen auf der volkswirtschaftlichen Ebene eine wichtige, aber keine ausreichende Perspektive. Sie ist Ausdruck eines Verständnisses, regionale Entwicklung unabhängig von der makroökonomischen Situation zu sehen und reflektiert das weit verbreitete Verständnis der 1990er Jahre, in welchen Raumentwicklung vorrangig als global-lokales Phänomen verstanden wurde. Damit wird eine Realität suggeriert, in der Regionen unabhängig von der Verschuldung des Staates, der Qualität öffentlicher Verwaltung und ,makroökonomischer Arrangements“ (wie Lohnpolitik, Steuersystem u. Ä.) gedeihen. Es ist die zentrale These dieses Beitrages, dass sich die regionalen Disparitäten Europas in den 2010er Jahren allein durch die regionale Sichtweise nicht adäquat erfassen lassen.

Für diesen Beitrag lassen sich zwei miteinander verbundene Fragestellungen formulieren: Erstens soll allgemein der Zusammenhang zwischen der Entwicklung

\footnotetext{
1) PIIGS: Eine während der Euro-Krise entstandene und gängig gewordene Abkürzung für die krisenhaften Euroländer Portugal, Italien, Irland, Griechenland und Spanien.
} 
nationaler und regionaler Disparitäten oder der Einfluss der makro-ökonomischen Ungleichgewichte in der Eurozone auf die regionalen Disparitäten der betreffenden Länder untersucht werden. Zweitens soll der Frage nachgegangen werden, wie sich die gegenwärtige Banken-, Staatsschulden-, Euro- und Wirtschaftskrise auf die regionalen Disparitäten innerhalb der Länder der Eurozone auswirkt. Die Intention dieser Arbeit ist es, die Bedeutung volkswirtschaftlicher Strukturen und Entwicklungen für die regionale Entwicklung aufzuzeigen. Dazu gliedert sich der Text wie folgt: Nach einer theoretisch-konzeptionellen Diskussion der Region als Perspektive der europäischen Raumentwicklung (Kapitel 2) erfolgt eine Auseinandersetzung mit den regionalen Disparitäten in Europa (Kapitel 3). Sodann werden die nationalen Disparitäten vor dem Hintergrund der Eurozone (Kapitel 4) sowie die regionalen Disparitäten (Kapitel 5) im nationalen Kontext analysiert. Abschließend erfolgt eine Diskussion der Ergebnisse.

\section{Region und Staat als widersprüchliche europäische Raumparadigmen}

Das räumliche Selbstbild der Europäischen Union und ihrer Vorläuferorganisationen (Europäische Gemeinschaft für Kohle und Stahl, EGKS; Europäische Wirtschaftsgemeinschaft, EWG; Europäische Gemeinschaften, EG) ist von einem Mosaik von Regionen und Städten bestimmt, visualisiert in Raumentwicklungsmodellen wie etwa der „Blauen Banane“ Brunets (Kunzmann \& Wegener 1991), dem Pentagon (ESPON 2004) oder der „Traubenstruktur“ (Kunzmann 1992). In keinem dieser Modelle sind Staatsgrenzen dargestellt: Die räumliche Entwicklung Europas wird als ein Prozess gesehen, für den die Existenz von Nationalstaaten obsolet geworden zu sein scheint. Dieser Zugang ist das Ergebnis eines Zusammenspiels zweier Faktoren: einerseits der institutionellen und ideellen Aufwertung der Region als Handlungsebene der europäischen Politik und andererseits der Durchsetzung eines raumwissenschaftlichen Paradigmas, in dem regionale und urbane Strukturen als zentrale Motoren und Bühnen der wirtschaftlichen, technologischen und sozialen Entwicklung - also der Globalisierung - verstanden wurden.

\subsection{Die Region als Baustein eines Vereinigten Europas}

Für die Gründung der EGKS im Jahr 1952 und später der EWG im Jahr 1958 waren geopolitische Ziele wie die Eindämmung des Kommunismus, die Westintegration Deutschlands (SLOTERDIJK 2008) sowie die Sicherung des Friedens ausschlaggebend. Eng verknüpft damit waren wirtschaftliche Ziele, nämlich der Erhalt und die Vermehrung des Wohlstandes (Vorauer 1997; Hudson 2003). Die Verringerung regionaler Disparitäten war nicht zuletzt deshalb kein Ziel des frühen Integrationsprozesses, weil es sich um eine überwiegend homogene Staatengruppe handelte und da gemäß dem neoklassischen Paradigma (vgl. Cecchini-Report; Europäısche Kommission 1988) Wachstum und Integration als Voraussetzungen für eine konvergente räumliche Entwicklung gesehen wurden. 
Der Aufstieg der Region als Symbol und Paradigma im europäischen Integrationsprozess ist unter mehreren Aspekten zu sehen: Erstens nahmen mit einer zunehmenden horizontalen Ausweitung der Gemeinschaft die räumlichen Ungleichgewichte zu (Süderweiterung, Osterweiterung, vgl. DüHR 2013, S. 215); zweitens kam es durch die Vertiefung des Binnenmarktes keineswegs zu einer eindeutigen Konvergenz der regionalen Entwicklung. Es zeigte sich, dass die Strukturen und Erfolgsrezepte wettbewerbsfähiger Regionen nur bedingt verallgemeinerbar waren und als unerwartete Nebenwirkung auch Verliererregionen produziert wurden (EuROPÄIsche KoMmission 1999, S. 10f.). Drittens sollte die zunehmende politische Kompetenzverlagerung zu den europäischen Institutionen, wie etwa durch den Vertrag von Maastricht, legitimiert werden, indem man bestrebt war, die Schwächung der nationalstaatlichen Ebene durch eine Stärkung der regionalen Ebene zu kompensieren - dies im Sinne des Subsidiaritätsprinzips und einer ausgeprägteren Bürgernähe des europäischen Integrationsprojektes (PAAsi 2001, S. 14f.). Letztlich entsprach diese Strategie auch einem gewissen Zeitgeist, der regionale Identität als Gegenmodell zu globalen Entankerungsprozessen und einer stärkeren Bürgerpartizipation entwarf (Hudson 2003, S. 57).

Diese politics of scales der Aufwertung der Regionen verdeutlicht sich in der Hochkonjunktur des Schlagwortes „Europa der Regionen“ sowie in der Verankerung im europäischen Institutionenapparat (KEATING 1997). So kam es bereits 1975 (Einführung des EFRE) zu einer Ausweitung der Gemeinschaftspolitik auf die nationalen Regionalpolitiken, die sukzessive weiter ausgebaut wurde (etwa die Reform der Strukturfonds 1988; VORAUER 1997, S. 31). Weitere Schritte der Institutionalisierung bildeten die Konferenz „Europa der Regionen“ (1989) sowie der „Ausschuss der Regionen“ (1992), die allerdings die Hoffnung der Regionen auf stärkere Mitbestimmung nicht erfüllen sollten. Im Jahr 1999 veröffentlichte die Kommission das Europäische Raumentwicklungskonzept (EUREK), in dem das territoriale Kohäsionsziel in der EU explizit formuliert wurde (EuropÄIsche Kommission 1999, S. 7). Zeitgleich mit dieser institutionellen Verankerung der Region als Handlungsebene der europäischen Politik fand ein Paradigmenwechsel in den Raumwissenschaften statt.

\subsection{Die ,regionale Brille“ als Paradigma in den Raumwissenschaften}

Auch wenn die Aufwertung der Region in den Institutionen der Europäischen Union häufig nur rhetorischer und symbolischer Natur war, so entsprach sie auch einem Paradigmenwechsel der Sozialwissenschaften in den 1990er Jahren. Symptomatisch dafür waren die sogenannten „De-Territorialisierungstheorien“, die den Staat sowohl als räumliche Analyseebene, als auch als relevanten Akteur für ökonomische und soziale Prozesse ablehnten (als zusammenfassende Kritik vgl. KeIL \& Brenner 2003 sowie ZeLLER 2003). Die Krise des keynesianistischen Wohlfahrtsstaates und der Wandel der fordistischen Produktionsstrukturen auf der einen (Jessop 1997, S. 60) sowie die Durchsetzung neuer globaler Produktions- und Organisationsstrukturen auf der anderen Seite verlangten nach neuen Ansätzen, die die ,territoriale Falle“ des „,nationalstaatlichen Macht-Containers“ überwinden sollten (ANGEw 1994). Die GlobalCity-Theorie von Saskia SASSEN (1991) und der Space-of-Flows-Ansatz von Manuel Castells (1998) verstanden städtische Entwicklungen als Prozess, der zwischen der 
lokalen und globalen Ebene erfolgt. Der Begriff der „Glokalisierung“ (RoBERTSON 1995) verdeutlicht, welch geringe Bedeutung dem Staat im Rahmen räumlicher Entwicklungsprozesse beigemessen worden ist.

Auch die Wirtschaftsgeographie hat das regionale Paradigma aufgegriffen. Zahlreiche empirische und theoretische Arbeiten haben sich mit der Analyse regionaler Produktionsstrukturen, der Embeddedness von Unternehmen in Clustern (PORTER 1999) und Milieus (FromHold-EISEBith 1995) sowie der räumlichen Organisation von Unternehmens- und Wertschöpfungsketten (zum Überblick vgl. FISCHER et al. 2010) auseinandergesetzt. Häufiges Ziel dieser Arbeiten war zumindest implizit die Frage, Erfolgsfaktoren ökonomisch prosperierender Regionen zu identifizieren und daraus verallgemeinerbare Erfolgsrezepte abzuleiten. Damit kam es auch zu einem Wandel des Begriffs der Region: In den 1980er Jahren verstand man darunter noch ein nachhaltiges Gegenmodell mit einer partiellen Abkoppelung von der Globalisierung und der kapitalistischen Weltwirtschaft (vgl. Heintel 1998, S. 45f.), hingegen stand in den 1990er Jahren die erfolgreiche Integration in globale Wissens- und Produktionsnetzwerke im Vordergrund. Die Region als Cluster, Global City Region oder regionales Milieu waren eng mit dem Konzept der Wettbewerbsfähigkeit verknüpft und fügten sich somit auch nahtlos in das neoliberale Paradigma der Europäischen Union.

\subsection{Staatliche Strukturen und der Einfluss auf die räumliche Entwicklung}

Mit dem „Ende der Globalisierungsparty“, die ihren Höhepunkt in den 1990er Jahren erlebte (PECK 2000, zitiert aus KeIL \& BREnNer 2003, S. 261), begann sich in den 2000er Jahren langsam die Erkenntnis einzustellen, dass in den Sozialwissenschaften mit den De-Territorialisierungstheorien möglicherweise das Kind mit dem Bade ausgeschüttet worden war (KEIL \& BRENNER 2003, S. 261). Der Staat schien als mächtiger Akteur nicht Einfluss verloren zu haben, vielmehr kam es durch die Re-Territorialisierung zu einer Neukonfiguration und Maßstabsverschiebung territorialer Macht und Einflussnahme (BRENNER 2004). Zahlreiche Beispiele wie der Thatcherismus für London (LEYSHON \& THRIFT 1997; ZEHNER 2011) oder die deutsche Volkswirtschaft (und Wirtschaftspolitik) für Frankfurt am Main (vgl. Grote 2003; Schamp 2011) zeigen, dass Staaten durchaus eine starke Rolle bei der Formation ,ihrer“ Global Cities spielten. In diesem Sinne hat auch Saskia SASSEN in einer jüngeren Publikation darauf hingewiesen, dass die Global City nicht ausschließlich durch globale Prozesse, sondern auch aus den Institutionen und Strukturen des Staates und seinen historisch-territorialen Logiken heraus zu erklären ist (SASSEN 2006, S. 4).

Global Cities werden also nicht nur durch globale Verflechtungen und lokale Netzwerke sowie Agglomerationsvorteile, sondern auch - im BouRdiEUschen Sinne - durch den Staat ,produziert“. Führt man dieses Argument über die Global CityDebatte hinaus und überträgt es auf die räumlich-regionale Entwicklung, so lassen sich verschiedene Einflusssphären des Staates (beispielhaft und ohne Anspruch auf Vollständigkeit) anführen: 
- Der Einfluss der räumlichen Staatsorganisation auf die optimale Allokation öffentlicher Güter ist ein klassisches Thema der Föderalismusforschung (Musgrave et al. 1992; Lijphart 1999; Qian \& Weingast 1997), die in den letzten Jahren auch dem Einfluss dezentraler Staatsstrukturen auf die Entwicklung räumlicher Disparitäten nachgegangen ist. Einerseits schaffen dezentrale Systeme (wie in Deutschland, Österreich oder den USA) einen gewissen Wettbewerb und Effizienzvorteile zwischen Gebietskörperschaften, andererseits erhöhen die regionalen fiskalischen Spielräume die Unterschiede zwischen armen und reichen Gebietskörperschaften. Dennoch überwiegen Studien, die einen positiven Einfluss dezentraler Strukturen auf die regionale Konvergenz bestätigen (Shankar \& Shah 2003; Lessmann 2009, S. 2471), wobei dieser Zusammenhang für wohlhabende Staaten eher gilt als für ärmere, die keine regionalen Ausgleichsmechanismen finanzieren können. So hat der Finanzausgleich, der in Österreich zwischen den Gebietskörperschaften jährlich rund $13 \mathrm{Mrd}$. Euro umverteilt (SCHRATZENSTALLER 2005), einen wichtigen, wenngleich bislang wenig untersuchten Einfluss auf die räumliche Entwicklung. Für die europäischen Staaten zeigt sich, dass dezentrale Systeme eine stark dämpfende Wirkung auf regionale Disparitäten haben, während in zentralen Staatsorganisationen divergierende Trends überwiegen (Musil 2012, S. 21).

- In entwickelten Industrieländern sind in der Ära des Fordismus unterschiedliche, spezifisch makro-ökonomische Arrangements, sogenannte „Varieties of Capitalism“ entstanden (vgl. Hall \& Soskice 2001; AmABLE 2003); als spezifische Faktoren sind hier vor allem die nationalen Arbeits- und Sozialrechte zu nennen, ebenso die Formen der Finanzierung von Unternehmen sowie die Marktkoordination durch staatliche Institutionen und die Politik (vgl. Hancké 2009). So können für Europa vier unterschiedliche makro-ökonomische Arrangements unterschieden werden: ein liberales angelsächsisches Modell, ein korporatistisch-rheinländisches Modell, ein korporatistisch-konsensuales skandinavisches Modell sowie ein peripheres, südliches Modell mit einer hohen öffentlichen Verschuldung (vgl. Hudson \& WiLliams 1999; Hudson 2003, S. 50). Ma \& Timberlake (2012, S. 2) unterscheiden bei makroökonomischen Arrangements auf einer globalen Ebene zwischen dem liberalen Laissez-faire-Typ und dem dirigistisch-interventionistischen Entwicklungstypus und zeigen auf, dass durch deren unterschiedliche Internationalisierungsstrategien die räumliche Entwicklung nationaler Städtesysteme sehr unterschiedlich erfolgt (ebd., S. 14f.).

- Ein zentrales Charakteristikum europäischer Staaten ist - etwa im Vergleich zu den USA - die Existenz großer und leistungsfähiger Sozialsysteme, die jedoch sehr unterschiedlich ausgeprägt sind. Die klassische Unterscheidung zwischen dem liberalen, konservativen und sozialdemokratischen Modell (EsPING-ANDERSEN 1990; KAUFMANN 2003) steht sicherlich in einem engen Konnex zum jeweiligen makro-ökonomischen Arrangement und beeinflusst das sozial-räumliche Gefüge, insbesondere das Ausmaß sozialräumlicher Polarisierung (KAZEPOv 2005, S. 16): Vor allem in europäischen Städten zeigen sich die in der Global-City-Theorie postulierten sozialen Spaltungen nur in sehr abgeschwächter Form (vgl. Hamnett 2003, S. 102; Häussermann \& Haila 2005). 
Die territorialen Organisationsstrukturen und ihre intergouvernementalen Umverteilungsmechanismen (Finanzausgleich), die Wohlfahrtssysteme und die makroökonomischen Arrangements der Staaten beeinflussen die räumliche Entwicklung und die regionalen Disparitäten abseits der schubladisierten „Regionalpolitik/-förderung“, deren Leistungsfähigkeit natürlich wieder stark von der Prosperität des Staates abhängt. Letztlich sind auch regionale Entwicklungsstrategien und -politiken das Ergebnis staatlicher Politik, in nationale Innovationsstrategien eingebettet und durch diese auch finanziert; von einem ,bypassing the nation-state“ (HEEg 1996, S. 214) kann hier also keine Rede sein. Damit liegt es nahe, die Frage der gegenwärtigen Wirtschaftskrise aus dem Blickwinkel der jeweiligen Staaten zu diskutieren.

Weiters verdeckt das Schlagwort des „Europas der Regionen“ den Umstand, dass die Stellung der Regionen innerhalb der Europäischen Staaten - hinsichtlich ihrer fiskalischen und legislativen Autonomie - sehr unterschiedlich ausgeprägt ist und damit zugleich auch ihre Einflussmöglichkeit, als mächtiger wirtschaftlicher oder sozialer Akteur zu wirken (vgl. PAAsi 2001, S. 15). Es fragt sich, ob der von Rem KoolhaAs aus einer durchaus kritischen Sicht entworfene Barcode (McNeILl 2006, S. 86), der sich aus den nationalen Flaggen der EU-Mitgliedsstaaten zusammensetzt, eine räumlichterritoriale Logik symbolisiert, die der räumlichen Struktur der Europäischen Union eher entspricht als die offizielle Flagge der EU (vgl. Abb. 1).

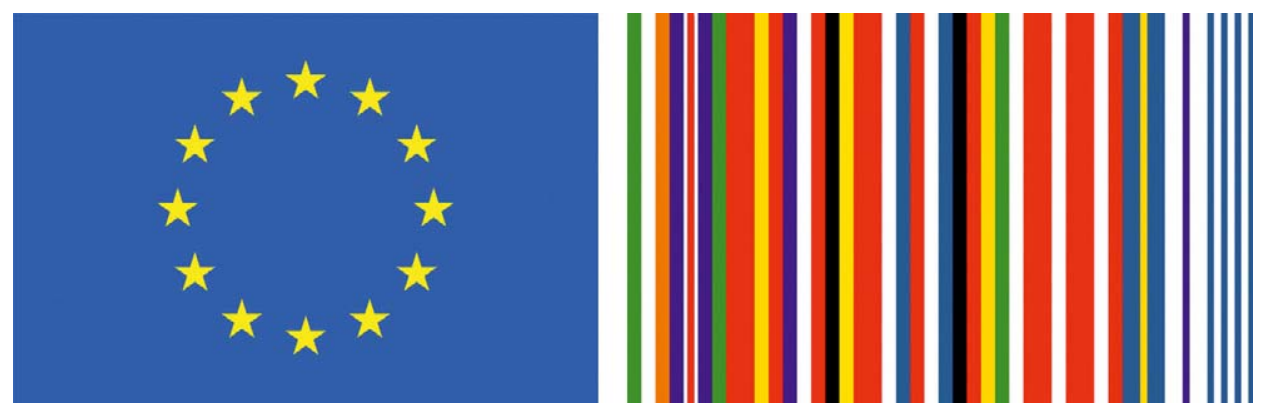

Quelle: EuropäIsche Kommission; KoOlhaAs 2002

Abb. 1: EU-Flagge und „barcode“ von Reem KoolHAAs als Ausdruck unterschiedlicher räumlich-territorialer Logiken

\section{Regionale Disparitäten in Europa - theoretische und empirische Befunde}

\subsection{Integration und Kohäsion - ein Widerspruch?}

Die Europäische Union wurde in den 1990er Jahren als Kohäsionsmotor oder als „catch-up machine“ (DunFord 2005, S. 157) charakterisiert, wofür zwei Umstände ausschlaggebend waren: erstens das verhältnismäßig geringe Wachstum von Regionen mit höherem Entwicklungsniveau und zweitens die Förderungen der Strukturfonds (vgl. 
ÖROK 2009, S. 180). Dennoch herrscht weder in theoretischer noch in empirischer Hinsicht Einigkeit in der Frage, ob es in der Europäischen Union zu einer Abnahme regionaler Disparitäten kommt. Die Standpunkte und Befunde der Konvergenz- und Divergenzthese können wie folgt zusammengefasst werden.

Vertreter der Konvergenzthese knüpfen an zwei zentralen Argumenten an (PETRAKOS et al. 2011): einerseits am neoklassischen Wachstumsmodell, das von konstanten Skalenerträgen, einem abnehmenden Grenznutzen des Kapitals und der Substituierbarkeit der Produktionsfaktoren ausgeht und bei freier Mobilität der Produktionsfaktoren die Konvergenz in einem Gleichgewichtspunkt annimmt (als Überblick vgl. ScHÄTZL 2011; Palme \& Musil 2012, S. 215ff.); zweitens an der neoklassischen Handelstheorie (Heckscher-Ohlin-Theorem), die aufgrund komparativer Vorteile zu gegenseitigen Handelsgewinnen und bei vollständiger Kapitalmobilität zur Konvergenz der Preisniveaus führt. Zahlreiche empirische Studien belegen für sehr unterschiedliche Zeiträume (alle zwischen den Jahren 1950 und 2000) eine Beta-Konvergenz ${ }^{2)}$, wenngleich bezüglich der Geschwindigkeit der Konvergenz unterschiedliche Ergebnisse vorliegen (vgl. GepPert et al. 2005; als Überblick Petrakos et al. 2011, S. 377) und überwiegend die geringe Geschwindigkeit (2\% Konvergenzrate) des Aufholprozesses sowohl für die USA wie auch für Europa betont wird (insbesondere BARRo \& SALAi-Martin 1991; Sala-i-Martin 1996, S. 1339; Boldrin \& Canova 2001, S. 37). Den allgemeinen Konvergenztrend, den SALA-I-MARTIN für Europa und die USA zwischen 1950 und 1990 festgestellt hat, ist für ihn auch Ausdruck eines geringen Einflusses der nationalen Politik. Dies insbesondere deshalb, da sich die Bemühungen zur konvergenten Raumentwicklung von Land zu Land sehr unterscheiden, die von ihm bestimmte Konvergenzrate von $2 \%$ hingegen kaum Unterschiede zwischen den Ländern zeigt (SAla-i-Martin 1996, S. 1341f.).

Demgegenüber knüpfen die Befürworter der Divergenzthese an verschiedenen polarisationstheoretischen Ansätzen an - wie etwa der sektoralen Polarisation von PerRoux oder der regionalen Polarisation von Myrdal (vgl. Schätzl 2011) -, die Wachstum als regional konzentrierten und ungleich ablaufenden Prozess verstehen. Neben der endogenen Wachstumstheorie (ROMER 1986) betont auch als jüngerer Ansatz die neue Wachstumstheorie (KRUGMAN 1991) die Bedeutung von steigenden Skalenerträgen, monopolistischen Marktstrukturen und räumlich differierenden Transportkosten, die zu einem räumlich konzentrierten Wirtschaftswachstum führen. Auf den europäischen Integrationsprozess übertragen ist demnach davon auszugehen, dass Regionen aufgrund ihrer unterschiedlichen Faktorausstattung und Agglomerationsvorteile vom steigenden Wettbewerb des Binnenmarktes sehr unterschiedlich profitieren. Auch diese theoretische Einschätzung wird durch jüngere empirische Studien unterstützt, die eine unterschiedliche Zahl (vier oder fünf) an „Konvergenzclubs“ identifiziert haben, also Typen von Regionen, die einander ähnlicher werden, während die Divergenzen zwischen diesen Gruppen zunehmen (vgl. QUAH 1996; RoDRIGUEZ-Pose 1999 oder CANOva 2004).

2) Beta-Konvergenz liegt vor, wenn periphere Regionen durch überdurchschnittliches Wachstum eine nachholende Entwicklung vollziehen; Alpha-Konvergenz meint die Abnahme der Streuung über die Zeit (vgl. Musil 2012, S. 8f.). 
Die widersprüchlichen Befunde zur Entwicklung räumlicher Disparitäten in der Europäischen Union zeigen, dass hier unterschiedliche Trends der Konvergenz und Divergenz wirken, wobei PETRAKos et al. (2011, S. 378) zusammenfassend festhalten, dass dies neben dem Entwicklungsniveau auch eine Frage der Maßstäblichkeit ist. Demnach überwiegen auf der europäischen Ebene konvergierende, auf der nationalen Ebene divergierende Entwicklungen. Diese widersprüchlichen Tendenzen der Raumentwicklung führen die Autoren auf die metropolitanen Regionen zurück (ibd., S. 378), da diese aufgrund ihrer starken überregionalen Verflechtung eine gewisse Entkoppelung von den jeweiligen Volkswirtschaften vermuten lassen.

\subsection{Die Bedeutung der europäischen Großstädte als „Wachstumsmaschinen“}

Mit dem Konzept der europäischen Metropolregionen wurden die großen Städte auch in der Raumplanung wieder stärker in den Fokus gerückt (vgl. KNIELING 2009; Blotevogel 2010). Bewertet man dieses Konzept vor dem Hintergrund der Lissabonstrategie (später Europa-2020-Strategie), deren Innovations- und Wachstumsorientierung auch in den Zielkatalog der EU-Regionalpolitik eingeflossen ist, handelt es sich dabei durchaus um ein Konzept, das die Neuorientierung vom Ausgleichs- zum Wachstumsgedanken mitvollzieht (VORAUER-Mischer 2009, S. 184). Somit haftet dem Konzept der Metropolregionen durchaus auch etwas Zeitgeistiges an (KNIELING 2009, S. 1f.) sowie auch die Hoffnung, dass durch Diffusions- und Ausbreitungseffekte auch periphere Regionen von der Dynamik der Wachstumskerne profitieren werden.

Für die Fokussierung auf Städte als Kerne eines wissensintensiven, innovations- und wachstumsorientierten Europas spricht eine Reihe wichtiger, miteinander verbundener theoretischer Argumente:

- Erstens existieren Agglomerationsvorteile (etwa durch die Existenz vielseitiger und qualifizierter Arbeitsmärkte, hohe Konsumentenkaufkraft, Nähe zu konkurrierenden und kooperierenden Unternehmen), die zu einer steigenden Produktivität und sinkenden Transaktionskosten (vgl. KujATH 2009, S. 204) bei zunehmender Größe und Dichte führen. Gemäß der „,density-competition effects“ (DiJkstra et al. 2013, S. 336) ist davon auszugehen, dass die sektorale Diversität sowie die Produktivität und damit auch das Wirtschaftswachstum in größeren Städten höher sind als in kleineren (vgl. für die USA Melo et al. 2009).

- Zweitens erleichtert eine hohe Dichte die Bildung von regionalen Wissensnetzwerken, funktionalen Verflechtungen von Unternehmen in Stadtregionen (LÜTHI et al. 2010) sowie die Ausbildung lokaler, kreativer Milieus (vgl. Fromhold-Eisebith 1995). Aus dieser Perspektive ist es nicht verwunderlich, dass die Innovationsfähigkeit von Volkswirtschaften häufig in deren Agglomerationen konzentriert ist, ebenso sind wissensintensive Cluster (vgl. PORTER 2000) vorrangig in Städten zu finden.

- Letztlich spielen die großen Städte eine wichtige Rolle als Knoten im Global CityNetzwerk. Es sind jene Städte, die als Markt- und Produktionsorte für jenes hochgradig spezialisierte Wissen dienen, das Unternehmen für den Aufbau und zur Steuerung ihrer globalen Standort- und Lieferverflechtungen benötigen (SASSEN 
1991). Dieses Wissen wird von spezialisierten sowie räumlich stark konzentrierten, unternehmensorientierten Dienstleistungsunternehmen bereitgestellt (TAYLOR 2004). Da für dessen Produktion Face-to-Face-Kontakte und lokale Wissensbeziehungen notwendig sind, ist dieses Wissen immobil und auf wenige Orte mit globaler „Connectivity“ konzentriert. Aus diesem Grund sieht SASSEN (1991, S. 8f.) eine „systemische Diskontinuität“" zwischen der Wirtschaftsentwicklung der Global Cities und der jeweiligen Staaten.

Aus diesen theoretischen Annahmen lässt sich die These ableiten, dass die europäischen Stadtregionen/Metropolregionen (1) ein überdurchschnittliches Wirtschaftswachstum aufweisen und (2) aufgrund ihrer starken internationalen oder globalen Vernetzung von den nationalen Ökonomien entkoppelt sind. Vor diesem Hintergrund ist es bemerkenswert, dass verschiedene empirische Befunde die These der systemischen Diskontinuität urbaner Ökonomien nicht stützen.

So hat Gornig (2004, S. 390) aufgezeigt, dass die Agglomerationsräume in der EU12 zwischen 1988 und 2000 eine unterdurchschnittliche Beschäftigungsentwicklung aufwiesen. In einer aktuelleren Studie haben Dijkstra et al. (2013, S. 342) gezeigt, dass überwiegend rurale Regionen in der EU-15 (jedoch nicht in den MOEL-Staaten) ein überdurchschnittliches Wirtschaftswachstum verbuchen konnten.

Neben der schwachen ökonomischen Performance haben die Studien jedoch anhand ausgewählter Agglomerationsräume auch gezeigt, dass die beträchtliche Heterogenität der städtischen Entwicklungspfade zumindest zum Teil auf ,die starken nationalen und historisch gewachsenen regionalen Prägungen“ (GoRNIG 2004, S. 395) zurückzuführen ist. In einem ESPON-Projekt wurden die Bedeutung oder der Einfluss der nationalen Wachstumsunterschiede für europäische Metropolen mittels Varianzanalyse geschätzt. Diese lag beim BIP/Kopf in den EU-12 bei 78\% (ESPON 2010, S. 44-45). Wenn auch innerhalb der Staaten zwischen Städten unterschiedliche Entwicklungstrends existieren, so unterstreichen die Studien die Bedeutung der nationalen Einflüsse auf die Entwicklung der urbanen Zentren.

Der Zusammenhang zwischen nationaler und regionaler Entwicklung, insbesondere vor dem Hintergrund der aktuellen, multiplen Wirtschaftskrise, scheint sowohl aus der Perspektive der regionalen Disparitäten als auch der urbanen Zentren wesentlich und bedarf angesichts der aktuellen makro-ökonomischen Ungleichgewichte der Eurozone einer weiteren empirischen Analyse.

\section{Konvergenzen und Divergenzen der Volkswirtschaften in der Eurozone}

Makro-ökonomische Ungleichgewichte zwischen Volkswirtschaften sind ein globales Phänomen, das sich in den Leistungsbilanzsalden widerspiegelt. So verfügen die USA mittlerweile seit Jahrzehnten über eine negative Leistungsbilanz, während Schwellenländer, allen voran China, seit den späten 1990er Jahren kontinuierlich positive Leistungsbilanzen erwirtschaften (IMF 2011). Weltbank und Währungsfonds 
weisen regelmäßig auf das Problem der „excessive imbalances“ hin, die die Stabilität der Weltwirtschaft gefährden. Die Ursachen dafür können (1) auf unterschiedliche Wachstumsraten (aufholende Staaten haben in der Regel einen Importüberschuss, wie die europäischen Transformationsländer), (2) manipulierte Wechselkurse (wie der niedrig gehaltene Kurs des Yuan), (3) ein Überangebot von Ersparnissen und Investitionskapital sowie (4) die unterschiedliche Wettbewerbsfähigkeit von Volkswirtschaften zurückzuführen sein (vgl. Breuss 2011, S. 414f.). Aufgrund der spezifischen Situation der Eurozone, die sich aus der gemeinsamen Währung ergibt, haben diese Faktoren unterschiedliche Bedeutung - vorrangig sind Unterschiede der Wettbewerbsfähigkeit und des Wirtschaftswachstums.

\subsection{Währungspolitische Integrationsbemühungen: EWS und Euro}

Die divergierenden Entwicklungen zwischen (derzeitigen) Eurostaaten haben eine lange Vorgeschichte. In der Zeit vor der Einführung des Euro existierten ebenfalls makro-ökonomische Ungleichgewichte, jedoch besaßen Staaten die Möglichkeit, negative Handelsbilanzen oder geringe Wettbewerbsfähigkeit durch Währungsabwertungen zu kompensieren. Dies zeigte sich am 1973 gegründeten Europäischen Währungssystem (EWS), das die Schaffung eines Wechselkurskorridors mit Abweichungen von $\pm 2,25 \%$ vom durchschnittlichen Kurs der Teilnehmerländer vorsah. Obwohl innerhalb des EWS beträchtliche Stützungskäufe zur Stabilisierung der Währungen stattfanden (vgl. Sinn 2012, S. 78), konnten die divergierenden Tendenzen nicht beherrscht werden. Vor allem Italien, dem eine Schwankungsbreite von $6 \%$ zugestanden wurde und welches mehrmals das EWS verließ, aber auch andere südeuropäische Staaten sowie das Vereinigte Königreich hatten mit den engen Schwankungsbreiten Schwierigkeiten, weil es sich um traditionell abwertungsgefährdete Währungen mit hoher Inflation und (aufgrund des Risikos) hohen Zinsaufschlägen auf den Kapitalmärkten handelte. Mit der Einführung des Euro im Jahr 1999 wurden die Währungsschwankungen schlagartig beendet, makro-ökonomische Ungleichgewichte artikulierten sich seither auf andere Weise: erstens durch Handelsbilanzen (die eben nicht mehr durch währung spolitische Maßnahmen beeinflusst werden können), zweitens durch Zinsaufschläge für Staatsanleihen, die das Vertrauen internationaler Investoren ausdrücken.

Die Zinssätze für zehnjährige Staatsanleihen zeigen eine Konvergenzphase seit der Euroeinführung (als offizielle Währung ab 1.1.2002) bis 2007 (vgl. Abb. 2). Mit dem Zusammenbruch des Interbankenmarktes infolge der US-Hypothekenkrise kam es zu einer neuerlichen Divergenz der Zinssätze: Die Homogenität der Eurozone wurde von Analysten stärker hinterfragt, womit auch die Angst vor einem Zahlungsausfall der ehemaligen Weichwährungsländer einherging. In beinahe der Hälfte der Eurozone - Griechenland, Portugal, Irland, Spanien, Italien (also den PIIGS-Staaten) sowie Zypern und Slowenien - stiegen die Zinssätze für zehnjährige Staatsanleihen, während diese in den restlichen (in Abb. 2 dargestellten) Staaten unter das Niveau von 2007 fielen. Nach einer langen Phase des sukzessiven Angleichens der Zinssätze bis 1997, spätestens 2002, reichte die Konvergenzphase bis in den Sommer 2007 und hatte trotz der vergleichsweise kurzen Dauer (maximal eine Dekade) weitreichende Auswirkungen, die erst durch die Eurokrise ab 2007 sichtbar wurden. 


\subsection{Effekte der Eurozone auf nationale Divergenzen}

Die Folge der Euroeinführung sowie des allgemein niedrigen Zinsniveaus in den Eurostaaten bedeutete für die südeuropäischen Staaten sowie für Irland eine Phase billigen Geldes - erstens, weil sich die Staaten zu sehr günstigen Konditionen an den Finanzmärkten verschulden konnten; zweitens, weil Anleger aus dem Norden der EU aufgrund des hohen Wachstums in der Peripherie investierten. Die niedrigen Zinsen wurden von den Staaten auch genutzt, um die Löhne in der Verwaltung überproportional ansteigen zu lassen (Griechenland nominell um 80\%, Portugal um 30\% zwischen 2000 und 2008) (SinN 2012, S. 87). Wachsende Einkommen im öffentlichen Sektor in Verbindung mit einer Zunahme der Privatkredite (auch für Haushalte fielen die Zinssätze) und einem wachsenden Interesse ausländischer Investoren lösten ein hohes, jedoch inflationsgetriebenes Wachstum aus.

Der durch das billige Geld ausgelöste Boom führte in den Ländern des Südens $\mathrm{zu}$ einem hohen Wirtschaftswachstum, sinkender Arbeitslosigkeit und steigenden Löhnen, womit sich die Lohnstückkosten insbesondere in Relation zu den ehemaligen Hartwährungsländern erhöhten. In Spanien bereiteten die niedrigen Zinsen auf dem Euro-Interbankenmarkt die Voraussetzung für einen Immobilienboom: Im Jahr 2005 wurden 812.000 Wohneinheiten errichtet, das sind 18.710 pro Mio. Einwohner; in Deutschland betrug der Vergleichswert lediglich 3.030 (vgl. Hasse 2010, S. 61). Ähnlich wie in den USA konnten die steigenden Immobilienpreise in Spanien und den anderen Boom-Ländern der Peripherie Europas (vgl. SinN 2012, S. 100) dazu verwendet werden, die Darlehensbeträge zu erhöhen und die gestiegene Darlehenssumme in den Konsum zu lenken, womit die Handelsbilanz dieser Staaten - ähnlich wie in den anderen Ländern der südlichen Peripherie - belastet wurde.

Hohe Leerstandsraten bei Immobilienbooms sind ein verlässlicher Indikator für das Platzen einer Blase (vgl. für die Asienkrise HusA \& WoHLschlägl 1999; allgemein vgl. HeEg \& Dörry 2009). In Spanien kündigten 750.000 bis 1.000 .000 leer stehende Wohnungen sowie über das ganze Land verstreute Geisterstädte im Jahr 2008 das Ende der Hausse an (HASSE 2010, S. 62). Die Immobilien- sowie die Wachstumsblase platzten, nachdem die Subprime-Krise in den USA auf die europäischen Banken übergegriffen hatte (vgl. Giese et al. 2011, S. 211f.) und die EZB den Leitzinssatz von 1,5\% (März 2006) auf 2,5\% (Dezember 2006) und weiter auf 3,75\% (Oktober 2008) anhob (vgl. Monatsberichte der EZB auf bundesbank.de). Mit dem Ende der Phase billigen Geldes endete auch das starke Wachstum in den ehemaligen Weichwährungsländern.

Das abrupte Ende der „Konvergenzdekade“ machte die volkswirtschaftlichen Ungleichgewichte, die durch die Zeit der niedrigen Zinssätze einerseits überdeckt, andererseits aber auch verstärkt wurden, schlagartig sichtbar. Im öffentlichen Sektor kam es in den PIIGS-Staaten zu einem Anwachsen der Staatsschulden, die aufgrund der negativen Ratings und steigenden Risikoaufschläge nur noch durch Hilfsmaßnahmen der EZB finanziert werden konnten. Der durch wachsende Lohnstückkosten gekennzeichnete und damit wenig wettbewerbsfähige Privatsektor erwirtschaftete eine negative Handelsbilanz, die sich ab 2007 in einer schrumpfenden Wirtschaft und steigender Arbeitslosigkeit manifestierte. Der Divergenz des Finanzsektors (vgl. Abb. 2) entsprach auch eine zunehmende Divergenz der makro-ökonomischen Fun- 


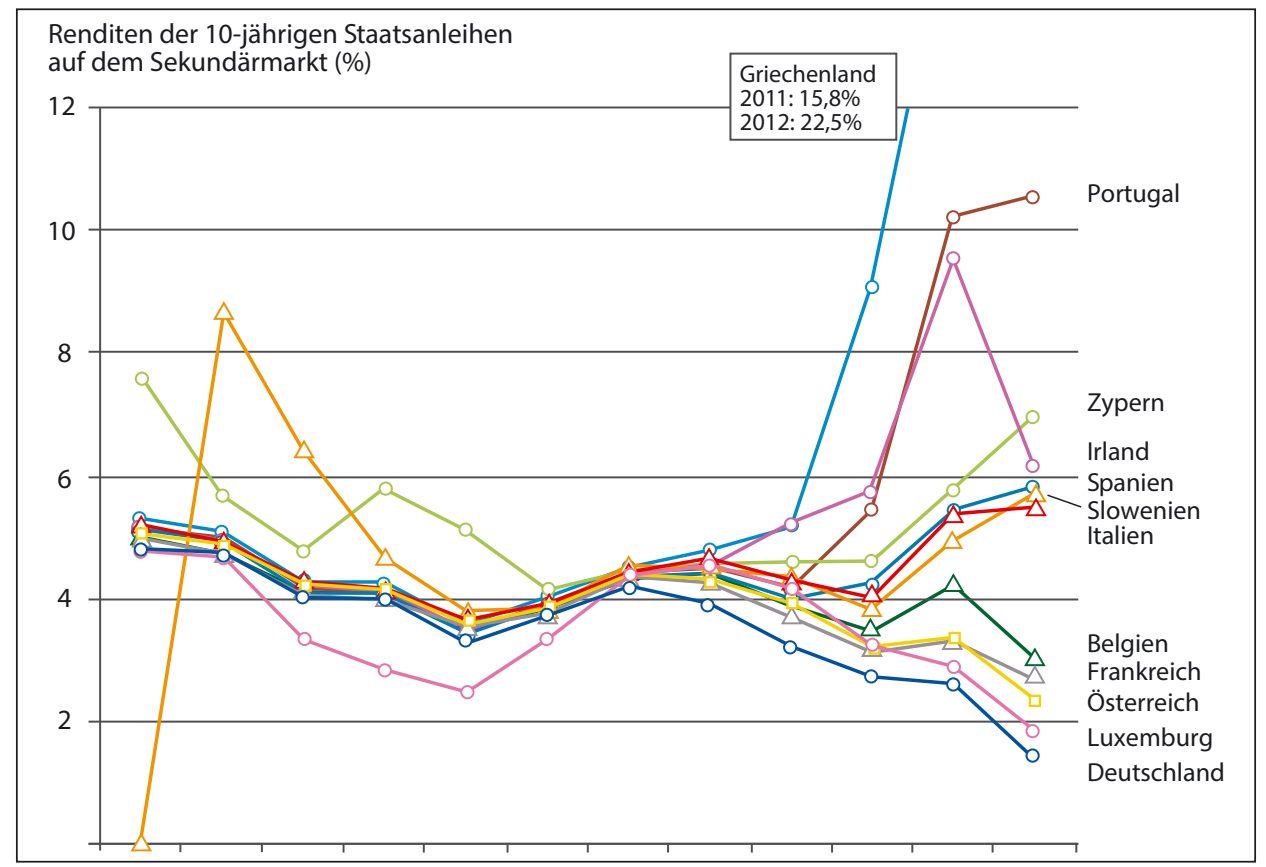

Quelle: Eurostat Database 2013

Abb. 2: Entwicklung der zehnjährigen Staatsanleihen auf dem Sekundärmarkt für ausgewählte Staaten der Eurozone

damentaldaten innerhalb der Eurozone ${ }^{3)}$, die sich im jährlichen Wirtschaftswachstum (BIP), den Arbeitslosenquoten sowie einer Schieflage der Leistungsbilanz (vgl. Tab. 1) niederschlägt.

Die Leistungsbilanzüberschüsse in den Zentrumsländern sind allerdings nicht, wie mitunter behauptet wird, die Folge ungleicher Handelsverflechtungen zwischen zentralen und peripheren Ländern (vgl. HADJimichalis 2011, S. 263), denn einerseits hat der Handel innerhalb der 14 hier berücksichtigten Eurostaaten seit 1999 kontinuierlich abgenommen, während Osteuropa, aber in noch höherem Ausmaß die aussereuropäischen Schwellenländer bei den Exporten der 14 Eurostaaten an Bedeutung gewannen (Eurostat Database 2013). Doch auch innerhalb der Eurozone gingen die positiven Handelsbilanzen der zentralen Länder nur zum Teil auf Kosten der (Semi) peripherie: Die Exporte in die peripheren Länder betrugen 2000 und 2004 rund 12\% (12,0 bzw. 12,1\%) und gingen mit dem Beginn der Krise, ab 2008, zurück (2012: $8,1 \%$; vgl. Eurostat Database 2013). Die steigenden Exportüberschüsse der sieben (ehemaligen) Hartwährungsländer wurden vorrangig (und mit steigender Tendenz) außerhalb der Eurozone erwirtschaftet.

\footnotetext{
3) Zur besseren Übersichtlichkeit der Darstellung wurden die Staaten in drei Gruppen eingeteilt, wobei die semiperipheren Staaten als „Indifferenzgruppe“ zu bezeichnen sind. Zentrum: Deutschland, Finnland, Luxemburg, Niederlande, Österreich; Semiperipherie: Belgien, Frankreich, Irland, Slowenien; Peripherie: Griechenland, Italien, Portugal, Spanien, Zypern.
} 


\begin{tabular}{|c|c|c|c|c|c|}
\hline & \multirow{3}{*}{$\begin{array}{r}1999 \\
3,70\end{array}$} & \multirow{3}{*}{$\begin{array}{c}2004 \\
3,04\end{array}$} & \multirow{3}{*}{$\begin{array}{r}\mathbf{2 0 0 8} \\
0,62\end{array}$} & \multirow{3}{*}{$\begin{array}{r}\mathbf{2 0 1 2} \\
-3,16\end{array}$} \\
\hline & & & & & \\
\hline \multirow{4}{*}{ 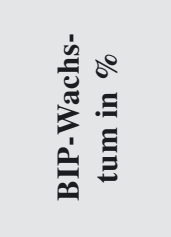 } & Peripherie & & & & \\
\hline & Semiperipherie & 5,80 & 3,65 & 0,55 & $-0,43$ \\
\hline & Zentrum & 4,48 & 2,90 & 0,78 & 0,12 \\
\hline & Varianz* & 6,19 & 1,40 & 2,55 & 4,06 \\
\hline \multirow{4}{*}{ 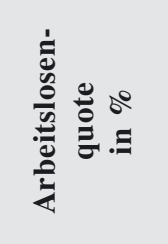 } & Peripherie & 10,28 & 8,30 & 7,58 & 17,56 \\
\hline & Semiperipherie & 7,95 & 7,13 & 6,40 & 10,35 \\
\hline & Zentrum & 5,72 & 6,86 & 5,14 & 5,58 \\
\hline & Varianz* & 12,15 & 5,63 & 4,99 & 44,67 \\
\hline \multirow{3}{*}{ 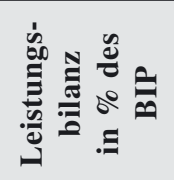 } & Peripherie & $-3,18$ & $-4,92$ & $-11,12$ & $-3,62$ \\
\hline & Semiperipherie & 1,18 & 0,13 & $-3,73$ & 0,88 \\
\hline & Zentrum & 2,92 & 6,52 & 4,68 & 4,48 \\
\hline
\end{tabular}

Quelle: Eurostat Database 2013

Tab. 1: Disparitäten in 14 Staaten der Eurozone (* Variationskoeffizient der Staaten)

\section{Regionale Disparitäten und makro-ökonomische Ungleichgewichte in der Wirtschaftskrise}

Angesichts der starken und zugleich hochgradig ungleichen makro-ökonomischen Entwicklung in der Eurozone ist die Frage nach der Entwicklung der regionalen Disparitäten innerhalb der Staaten relevant. Kommt es in den peripheren Staaten, die massiv unter der Krise leiden, zu einer Zunahme regionaler Disparitäten? Erfahren strukturschwache Regionen in jenen Staaten, die von der Krise weniger stark getroffen wurden, eher eine aufholende Entwicklung und lösen damit eine konvergente Raumentwicklung aus?

Regionale Disparitäten werden in der Regel auf zweierlei Arten bestimmt (vgl. Sala-I-Martin 1996, S. 1327f.): erstens durch die Alpha-Konvergenz, die sich in der Veränderung der Streuung einer Variable ausdrückt, etwa der Varianz ${ }^{4)}$ des Bruttoregionalproduktes je Einwohner, zweitens durch die Beta-Konvergenz, also das überproportionale Wachstum peripherer Regionen, die damit ein ,aufholendes Wirtschaftswachstum“vollziehen. Neben diesen beiden Messmethoden (vgl. Kap. 5.1 und 5.2) werden die urbanen Zentren der Staaten, die 40 Metropolen („Capital City Regions“ und „Second Tier Metro Regions“) gemäß Definition von Eurostat (vgl.

\footnotetext{
4) Der Vorteil der Varianz (etwa gegenüber der Standardabweichung) liegt darin, dass sie weder sensibel auf die Anzahl der Regionen noch auf die Höhe der Werte reagiert. Damit eignet sich die Varianz zum Vergleich unterschiedlich großer Volkswirtschaften, wie es in dieser Untersuchung der Fall ist (vgl. LESSMANN 2005, 28f.).
} 
Dijkstra 2009) und ihre ökonomische Performance im nationalen Kontext untersucht (vgl. Kap. 5.3). Damit kann geklärt werden, ob sich die großen Metropolen parallel zu den jeweiligen Staaten entwickeln oder ob diese vom nationalen Rahmen entkoppelt und damit von der Krise weniger betroffen sind. Lässt sich der von SALA-I-MARTIN (1996) für die Periode von 1950 bis 1990 festgestellte Pfad mit räumlich und zeitlich konstanten Konvergenzraten bestätigen oder verstärkt sich in der Krise eher der Trend zur Ausbildung von Konvergenzclubs?

\subsection{Alpha-Konvergenz: Nehmen regionale Ungleichgewichte ab?}

Innerhalb der zwölf Flächenstaaten der Eurozone (ohne Zypern und Luxemburg) mit insgesamt 172 Regionen $^{5)}$ haben die regionalen Disparitäten - gemessen am BIP/ Einwohner - seit 2000 deutlich abgenommen, wobei sich die Konvergenz auf die Jahre bis 2007 beschränkt; seither ist es wieder zu einer Zunahme der Disparitäten gekommen (vgl. Tab. 2). Zwischen den Staaten existieren jedoch beträchtliche Unterschiede, sowohl was das Niveau der regionalen Disparitäten als auch deren Veränderung betrifft. In den vier Staaten, die hier als Zentrum der Eurozone bezeichnet werden, ist es mit Ausnahme der Niederlande zu einer Abnahme der nationalen Disparitäten gekommen, wobei die konvergenten Entwicklungen nur den Zeitraum vor der Krise (bis 2007) betreffen. Seither ist es zu leichten Divergenzen (Deutschland, Finnland) oder zu einem Ende des Konvergenztrends (Österreich) gekommen. In den Niederlanden stagnierten die Disparitäten bis 2007 und nahmen seither deutlich zu.

Auch bei den als semiperipher bezeichneten Staaten lässt sich ein Trend zur regionalen Divergenz feststellen. Frankreich und das von der Krise besonders getroffene Irland verzeichnen in beiden Perioden zunehmende regionale Disparitäten, in Belgien flacht der konvergente Entwicklungstrend seit 2007 ab. Nur in Slowenien ist es durch die Krise zu einer Umkehr von einer deutlich divergenten zu einer konvergenten Raumentwicklung gekommen.

In den Staaten der Peripherie lässt sich ein ähnlich uneinheitliches Bild feststellen: In Italien und Spanien haben konvergierende Trends seit Ausbruch der Krise abgenommen, während in Portugal seit 2007 eine konvergente Raumentwicklung zu beobachten ist. In Griechenland nahmen die regionalen Disparitäten vor der Krise verhältnismäßig stark zu, nach der Krise flachte dieser Trend etwas ab.

In Summe zeigen die Varianz und deren Veränderung entgegengesetzte Trends in der Vorkrisen- und Krisenperiode, wobei es tendenziell in den meisten Ländern zu einer divergenten oder einer Abschwächung der konvergenten Raumentwicklung gekommen ist. Dabei scheint die Zentrum-Semiperipherie-Peripherie-Zuordnung, die ja auf den Zinsaufschlägen der Staatsanleihen und damit auf einer Bewertung durch Ratingagenturen basiert (vgl. Abb. 2), wenig Erklärungsgehalt für die jeweiligen nationalen Raumentwicklungstrends zu besitzen, denn zur deutlichsten Zunahme regionaler Disparitäten ist es in den vier Zentrumsländern gekommen.

5) In den zwölf Staaten der Eurozone wurden als räumliches Differenzierungsmerkmal überwiegend NUTS2-Regionen verwendet. In Irland und Slowenien wurden dazu die NUTS-3-Regionen herangezogen. 


\begin{tabular}{|c|c|c|c|c|c|}
\hline & \multicolumn{3}{|c|}{ Varianz } & \multicolumn{2}{|c|}{$\begin{array}{l}\text { Durchschnittliche jährliche } \\
\text { Veränderung der Varianz }(\%)\end{array}$} \\
\hline & 2000 & 2007 & 2010 & 2000-2007 & 2007-2010 \\
\hline Euro-12-Gruppe & 0,3467 & 0,3212 & 0,3248 & $-0,9$ & 0,3 \\
\hline \multicolumn{6}{|l|}{ Zentrum } \\
\hline Österreich & 0,2152 & 0,1959 & 0,1943 & $-1,1$ & $-0,2$ \\
\hline Deutschland & 0,2367 & 0,2178 & 0,2261 & $-1,0$ & 1,0 \\
\hline Finnland & 0,2439 & 0,2184 & 0,2389 & $-1,3$ & 2,3 \\
\hline Niederlande & 0,1698 & 0,1666 & 0,2056 & $-0,2$ & 5,8 \\
\hline \multicolumn{6}{|l|}{ Semiperipherie } \\
\hline Belgien & 0,4024 & 0,3696 & 0,3603 & $-1,0$ & $-0,6$ \\
\hline Frankreich & 0,1810 & 0,2025 & 0,2148 & 1,5 & 1,5 \\
\hline Irland & 0,2640 & 0,3022 & 0,3811 & 1,8 & 6,5 \\
\hline Slowenien & 0,1873 & 0,2370 & 0,2360 & 3,3 & $-0,1$ \\
\hline \multicolumn{6}{|l|}{ Peripherie } \\
\hline Spanien & 0,2247 & 0,1940 & 0,1940 & $-1,7$ & 0,0 \\
\hline Griechenland & 0,1625 & 0,2002 & 0,2155 & 2,9 & 1,9 \\
\hline Italien & 0,2673 & 0,2556 & 0,2556 & $-0,5$ & 0,0 \\
\hline Portugal & 0,2414 & 0,2462 & 0,2422 & 0,2 & $-0,4$ \\
\hline
\end{tabular}

Quelle: Eurostat Database 2013

Tab. 2: Regionale Disparitäten (BIP/Kopf) in den zwölf Eurostaaten zwischen 2000 und 2010

Deutlichere Aussagen zum Zusammenhang zwischen nationalstaatlicher Performance und regionaler Entwicklung lassen sich auf der Ebene der einzelnen Staaten machen. So existiert zwar kein klarer Zusammenhang zwischen dem Niveau der volkswirtschaftlichen Leistungsfähigkeit (BIP/Kopf) und dem Ausmaß der regionalen Disparitäten (Varianz des BIP/Kopf), allerdings lassen sich Zusammenhänge bei der Veränderung der beiden Variablen feststellen. Für den Zeitraum 2000 bis 2010 existiert ein (positiver) Einfluss des Wirtschaftswachstums auf die Veränderung regionaler Disparitäten.6) Differenziert man hingegen zwischen den Zeiträumen vor und nach dem Jahr 2007, so zeigen sich unterschiedliche Zusammenhangsmuster: Der bis 2007 positive Zusammenhang (vgl. Abb. 3, links) kehrt sich nach 2007 in einen negativen Zusammenhang (allerdings ohne signifikant zu sein; Abb. 3, rechts) um. Dies bedeutet, dass es durch das stagnierende oder negative Wachstum der Volkswirtschaften zu einer Zunahme der regionalen Disparitäten gekommen ist. Daraus ließe sich die interessante

6) Ein lineares Regressionsmodell hat zwischen dem Wirtschaftswachstum (unabhängige Variable) und der Veränderung regionaler Disparitäten (abhängige Variable) einen signifikant positiven Wert ergeben $\left(\mathrm{R}^{2}=38,8\right.$ Sig. $\left.0,031^{*}\right)$ 


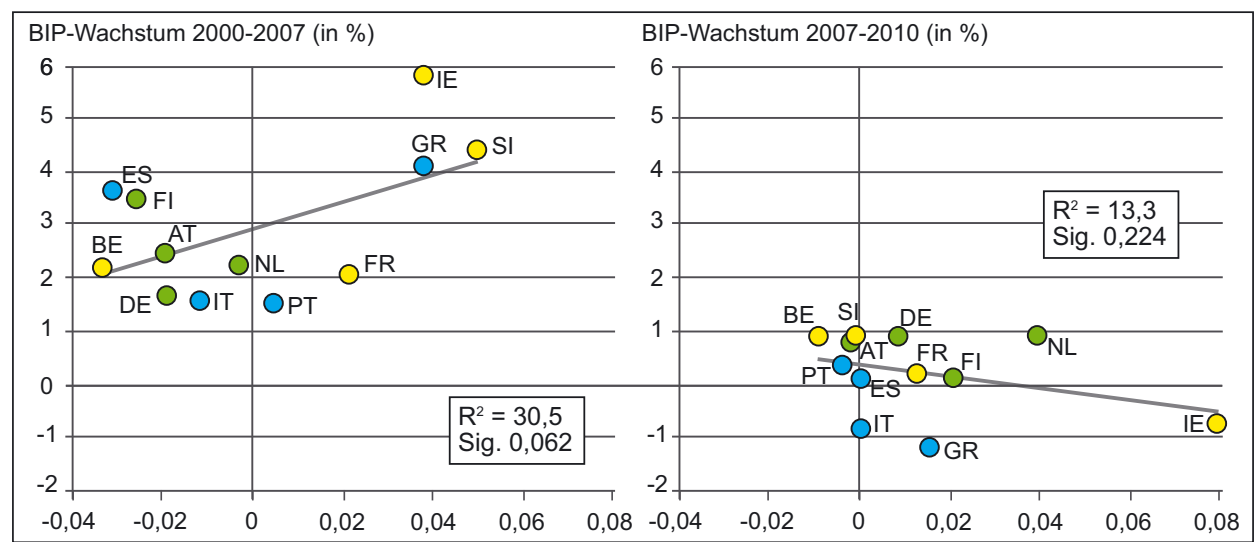

Quelle: Eurostat Database 2013

Abb. 3: Zusammenhang regionaler Disparitäten und makro-ökonomischer Performance (durchschnittliches jährliches BIP-Wachstum) in den zwölf Eurostaaten

These formulieren, dass tendenziell ein stärkeres Wirtschaftswachstum - egal ob mit positiven oder negativen Vorzeichen - zumindest kurzfristig zu steigenden regionalen Ungleichgewichten führt.

Die Darstellung der Varianz gibt zwar Auskunft über das Ausmaß der Streuung von NUTS-2-Regionen sowie deren Veränderung, es bleibt allerdings offen, ob etwa eine konvergente Raumentwicklung durch ein überproportionales Wachstum peripherer Regionen erzielt wurde oder ob zentrale Räume wirtschaftliche Prosperität verloren haben.

\subsection{Beta-Konvergenz: Holen periphere Regionen auf?}

\subsubsection{Entwicklungsniveau und nationale Beta-Konvergenz}

Die Beta-Konvergenz wird daran gemessen, ob periphere/rückständige Regionen gegenüber dem nationalen Durchschnitt überproportional wachsen. „Peripher“ oder „,semiperipher“ sind jene Regionen, deren BIP/Kopf in den beiden untersten Quintilen, gemessen an der Abweichung vom nationalen BIP/Kopf im Jahr 2000, liegt. Von den 172 Regionen sind demnach 70 als peripher oder semiperipher - im jeweiligen nationalen Kontext - einzustufen. In welchen der zwölf Staaten vollzogen diese Regionen Aufholprozesse und welche Wirkung hat die Wirtschaftskrise, insbesondere hinsichtlich der Stellung der Staaten innerhalb der Eurozone?

Einen Hinweis auf nationale Beta-Konvergenzprozesse liefert der Zusammenhang zwischen dem relativen Wirtschaftswachstum (Abweichung vom nationalen Wert) sowie dem relativen Wohlstandsniveau (Abweichung vom nationalen BIP/Kopf). In Summe, über alle 172 Untersuchungsregionen hinweg, zeigt sich hier ein positiver 
Zusammenhang, der jedoch vor der Krise schwächer war als danach (vgl. Tab. 3, Zeile 1). Es haben also tendenziell - seit 2007 deutlich mehr als zuvor - jene Regionen ein überdurchschnittliches Wachstum vollzogen, die im nationalen Kontext auch ein überdurchschnittliches Entwicklungsniveau aufweisen. Die zunehmende Stärke dieses Zusammenhanges belegt den empirischen Befund, dass durch die Krise regionale Disparitäten (also die Varianz, vgl. Tab. 2) zugenommen haben.

Differenziert man die 172 Regionen in die fünf Quintile (vgl. Tab. 3, Zeile 2 bis 6), so bestätigt sich dieses Zusammenhangsmuster. In der Periode 2000 bis 2007 bestand für periphere und semiperiphere (auch für mittlere) Regionen keinerlei Zusammenhang zwischen relativem Wirtschaftswachstum und relativem Entwicklungsniveau, d.h. auch periphere Regionen wuchsen im nationalen Kontext überdurchschnittlich. Zwischen 2007 und 2010 bestand hingegen ein positiver Zusammenhang oder es hat sich dieser in allen Regionstypen verstärkt. Der stärkste Zusammenhang existiert (für beide Perioden) bei den subzentralen Regionen. Die Wirtschaftskrise hat also dazu geführt, dass seit 2007 unterentwickelte Regionen tendenziell weniger in der Lage waren an die nationalen Durchschnittsniveaus anzuschließen.

\begin{tabular}{|l|c|c|c|}
\hline & $\mathbf{N}$ & $\mathbf{2 0 0 0}$ bis 2007 & $\mathbf{2 0 0 7}$ bis 2010 \\
\hline Gesamt & $\mathbf{1 7 2}$ & $\mathbf{0 , 3 7 9 * *}$ & $\mathbf{0 , 4 6 8 * *}$ \\
\hline Peripherie & 34 & 0,152 & $0,396^{*}$ \\
\hline Semiperipherie & 36 & 0,193 & $0,491 * *$ \\
\hline Mitte & 41 & 0,265 & $0,618 * *$ \\
\hline Subzentrum & 32 & $0,615^{* *}$ & $0,649 * *$ \\
\hline Zentrum & 29 & $0,480 * *$ & $0,637 * *$ \\
\hline
\end{tabular}

Quelle: Eurostat Database 2013

Tab. 3: Korrelationsmaße zwischen dem relativen Entwicklungsniveau (BIP/Kopf) und dem relativen Wirtschaftswachstum für die Perioden 2000 bis 2007 und 2007 bis 2010

\subsubsection{Makro-ökonomische Prosperität und Dynamik peripherer ${ }^{7)}$ Regionen}

Welchen Einfluss hat die ökonomische Prosperität einer Volkswirtschaft auf die nachholende Entwicklung peripherer Regionen? In der Periode vor der Wirtschaftskrise besteht ein eindeutig positiver Zusammenhang zwischen dem relativen BIP/KopfNiveau der zwölf Eurostaaten und dem relativen Wachstum der peripheren Regionen. Eine lineare Regressionsanalyse kann 48,5\% der Prosperität peripherer Regionen in den jeweiligen Staaten erklären (vgl. Abb. 4a). Demnach dürfte das ökonomische Entwicklungsniveau der Staaten einen beträchtlichen Einfluss auf die nachholende Entwicklung peripherer Regionen haben (etwa aufgrund leistungsfähiger wohlfahrts-/

\footnotetext{
7) Als ,peripher“ werden hier jene Regionen bezeichnet, die in Tabelle 3 die beiden unteren Quintile, also semiperiphere und periphere Raumeinheiten, umfassen.
} 

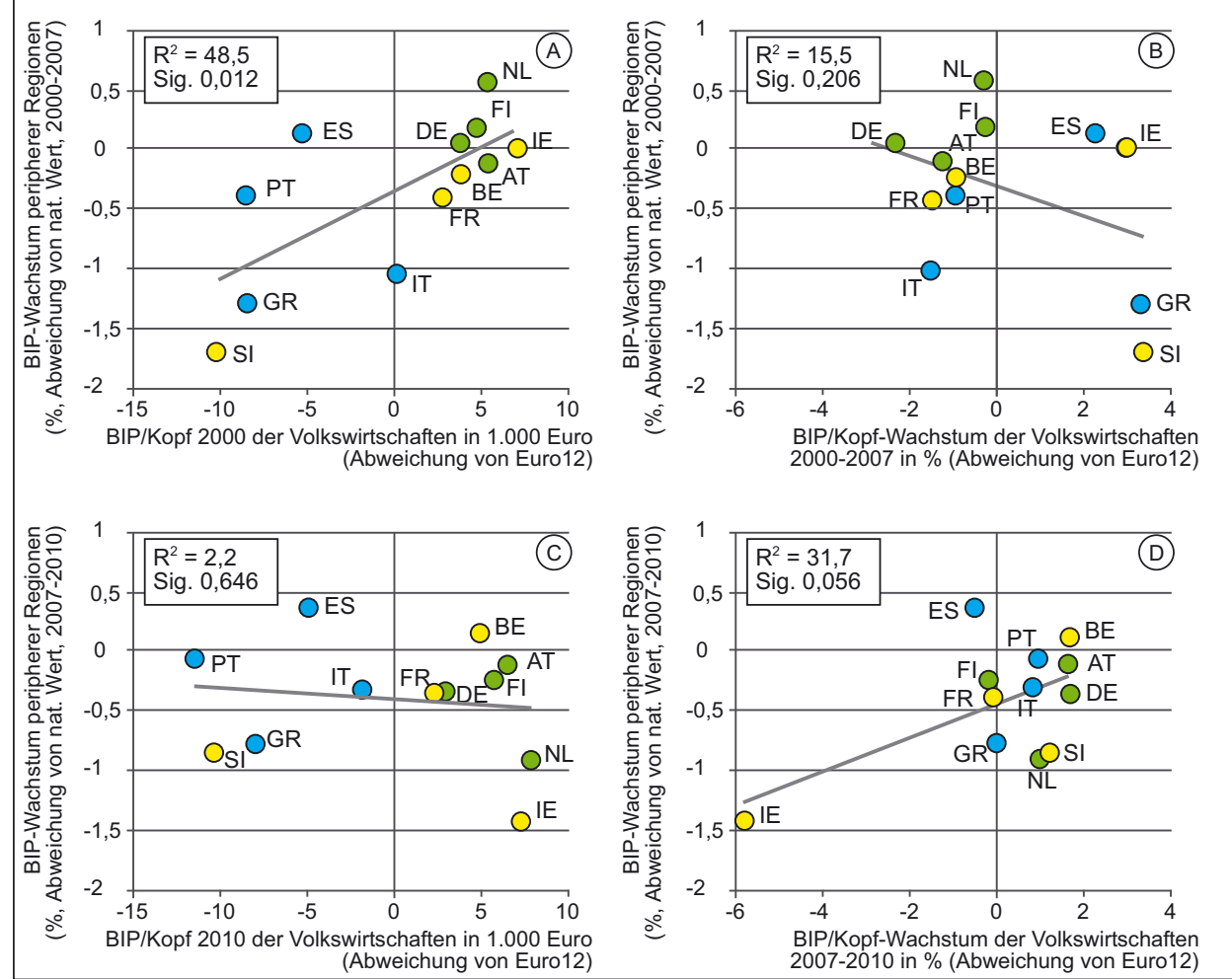

Quelle: Eurostat Database 2013

Abb. 4: Einfluss der makro-ökonomischen Performance (relatives BIP/Kopf in Abb. a/c sowie relatives BIP/Kopf-Wachstum in Abb. b/d) auf die Prosperität peripherer Regionen im nationalen Kontext

staatlicher Transfersysteme) - während dies für das Wirtschaftswachstum (relatives BIP/Kopf-Wachstum) nicht zu gelten scheint. Insbesondere in Griechenland und Slowenien waren periphere Regionen nicht vom nationalen Wachstumsboom erfasst, während bis 2007 in Staaten mit vergleichsweise geringem Wirtschaftswachstum (Niederlande, Finnland, Deutschland oder Österreich) gerade periphere Regionen ein nachholendes Wirtschaftswachstum verzeichneten (vgl. Abb. 4b).

Die Entwicklung seit 2007 (bis 2010) zeigt eine deutliche Veränderung des Verhältnisses zwischen nationaler und regionaler (peripherer) Prosperität (vgl. Abb. 4c). In den fünf Volkswirtschaften mit unterdurchschnittlichem BIP/Kopf-Niveau ist es seit Beginn der Krise zu einer vergleichsweisen Verbesserung der relativen Entwicklungsdynamik in den peripheren Regionen gekommen. Vermutlich scheinen die ökonomischen Zentralräume hier stärker von der Krise erfasst worden zu sein. Umgekehrt hat sich in Ländern mit überdurchschnittlichem BIP/Kopf-Niveau die Performance der peripheren Regionen verschlechtert. In Summe zeigt sich, dass der Einfluss des nationalen BIP/Kopf-Niveaus auf die regionale Entwicklung schwächer geworden ist. 


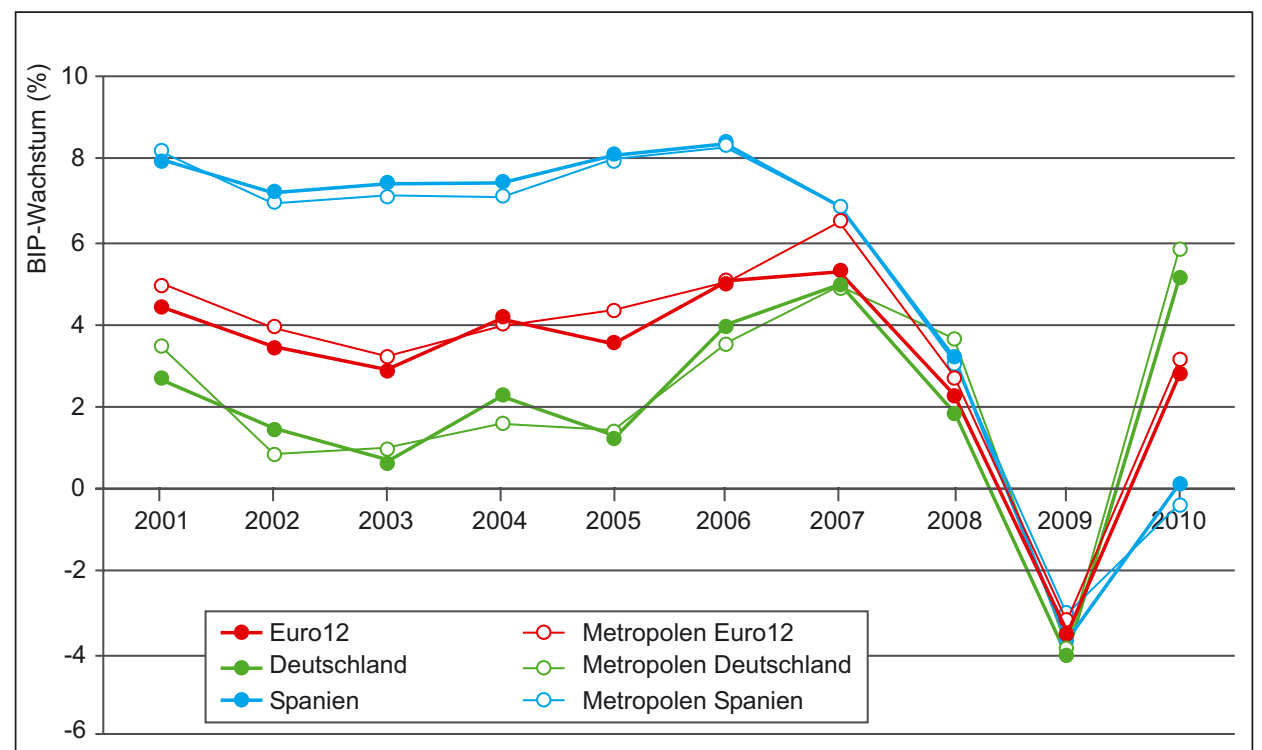

Quelle: Eurostat Database 2013

Abb. 5: Wirtschaftswachstum in Staaten der Eurozone (zwölf Territorialstaaten, Deutschland, Spanien) und deren Metropolregionen

Auch der Einfluss des relativen jährlichen BIP/Kopf-Wachstums hat sich seit 2007 grundlegend geändert (vgl. Abb. 4d): Es besteht ein positiver Einfluss (bei sehr geringer Signifikanz) auf das relative Wirtschaftswachstum peripherer Regionen, wobei der Ausreißer Irland zu berücksichtigen ist.

\subsection{Metropolen - Zugpferde der Euro-Volkswirtschaften?}

\subsection{1 Ökonomische Prosperität im volkswirtschaftlichen Kontext}

Die europäische Wirtschaftskrise hat auch die Metropolen in den Eurostaaten getroffen (Europäische Kommission 2013). In den zwölf Ländern haben die insgesamt 40 „Capital City Regions“ und „Second Tier Metro Regions“ (vgl. Dijkstra 2009), die hier als „Metropolen“ bezeichnet werden, seit 2007 massiv an Wirtschaftsdynamik verloren: Das durchschnittliche jährliche Wirtschaftswachstum sank von 3,41\% (2000 bis 2007) auf 0,26\% (2007 bis 2010). Um die jeweils nationalen Werte normiert, ging das relative Wirtschaftswachstum der Metropolen von 0,27\% auf 0,05\% zurück. Damit machen die Daten deutlich, wie eng das Wirtschaftswachstum der urbanen Zentren an jenes der jeweiligen Volkswirtschaften gekoppelt ist. Dies gilt sowohl für die zwölf Staaten in Summe als auch für einzelne Staaten, wobei die in Abbildung 5 dargestellten Beispiele Deutschland und Spanien idealtypisch die Kluft zwischen zentralen und peripheren Ländern verdeutlichen: Erstere wiesen vor der Krise ein deutlich niedri- 


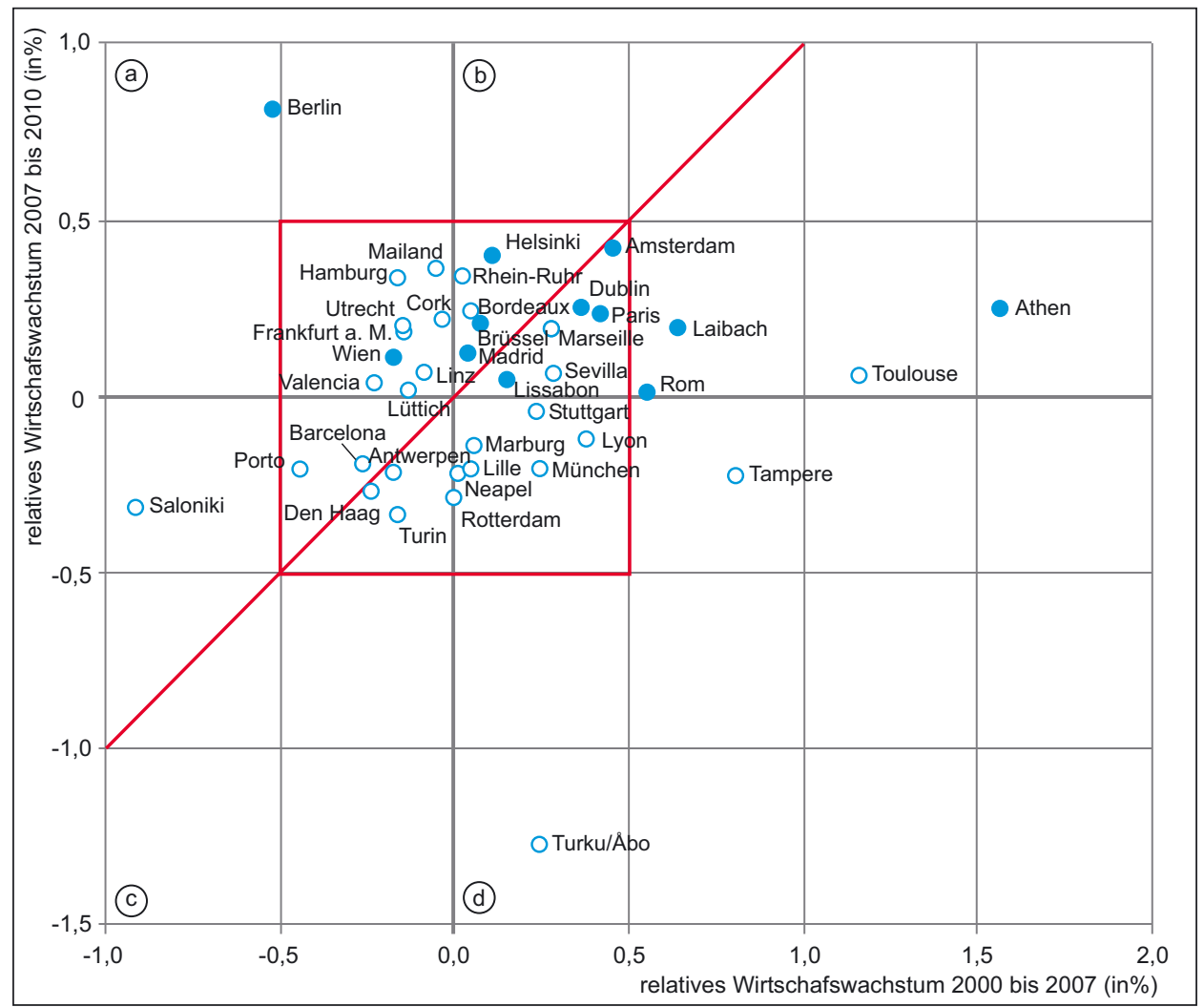

Quelle: Eurostat Database 2013

Abb. 6: Wirtschaftswachstum der europäischen Metropolregionen 2000 bis 2007 und 2007 bis 2012; Hauptstädte in Blau hervorgehoben

geres Wirtschaftswachstum auf, das ja insbesondere in Spanien, Griechenland oder Portugal durch eine inflationäre Geldpolitik induziert war; in den Krisenjahren seit 2007 konnten sich die zentralen Länder wesentlich rascher erholen. Diese nationalen Entwicklungstrends werden von den urbanen Zentren überraschend exakt nachvollzogen. Aus diesem Blickwinkel erscheint eine Entkoppelung der Metropolen von den volkswirtschaftlichen Wachstumspfaden als wenig wahrscheinlich.

Ein etwas differenzierteres Bild ergibt sich auf der Ebene der einzelnen Städte (vgl. Abb. 6). Von den 40 Metropolen hatten 15 sowohl in der Periode vor der Krise als auch während dieser ein überdurchschnittliches Wirtschaftswachstum erlebt (vgl. Abb. 6, Feld b). Weitere zehn Städte, die bis 2007 im nationalen Kontext stagnierten oder schrumpften, waren seit 2007 durch ein überdurchschnittlich starkes Wachstum charakterisiert (Feld a). Auffällig ist in diesen beiden Feldern, dass sämtliche Hauptstädte darin zu finden sind, wobei nur Berlin und Wien erst seit 2007 ein überdurchschnittliches Wachstum vollzogen. In den anderen zehn Hauptstädten lag das 
Niveau in beiden Perioden über dem nationalen Durchschnitt. Die Rolle der Stadt als „Wachstumsmotor“ scheint wohl eng mit dem „Hauptstadtbonus“ zusammenzuhängen. Unter der horizontalen Achse (Feld c und d) zeigen 15 Städte seit 2007 ein unterdurchschnittliches Wachstum, wobei der kleinere Teil (6 Städte) auch schon vor 2007 unter dem jeweiligen Landesdurchschnitt wuchs.

Wie für Deutschland und Spanien in Abbildung 5 zeigt sich auch hier, dass die Streuung des relativen Wachstums der Städte in einem relativ schmalen „Korridor“ stattfindet - nur acht Städte wichen um mehr als 0,5\% oder um weniger als $-0,5 \%$ vom jeweiligen nationalen Wirtschaftswachstum ab. In der Periode bis 2007 lagen nur sechs Städte außerhalb der $\pm 0,5 \%$-Schwankungsbreite (Saloniki [Thessaloniki], Rom [Roma], Laibach [Ljubljana], Tampere, Toulouse und Athen [Athina]), in der zweiten Periode sogar nur zwei, nämlich Berlin und Turku/Åbo. In Summe wächst die größere Gruppe der Städte leicht überdurchschnittlich, ist aber eng an die nationalen Dynamiken gekoppelt.

Eine Differenzierung der Städte nach dem zentral-peripheren Status der Staaten in der Euro-Zone (vgl. Kap. 5.2) erscheint aufgrund der Heterogenität in den drei Ländergruppen nicht sinnvoll: Eine Varianzanalyse hat für das relative Wirtschaftswachstum der beiden Perioden keine signifikanten Unterschiede feststellen können, umgekehrt ergaben sich signifikante Unterschiede zwischen den Wachstumsraten der zwölf Staaten. ${ }^{8)}$ Von einer Entkoppelung der urbanen Zentren von den nationalen Ökonomien (wie bei Petrakos et al. 2011 vermutet; vgl. Kapitel 3) hinsichtlich ihres Wirtschaftswachstums kann daher nicht gesprochen werden.

\subsubsection{Global-City-Status, Stadtgröße und ökonomische Performance}

Der Metropolisierungsgrad wird anhand zweier Dimensionen gemessen (vgl. KeMPER 2006, S. 3f.): erstens mittels der ,demographisch-strukturellen“ Dimension, worunter die Größe der Stadt nach ihrer Einwohnerzahl, ihre Dichte sowie die Monozentrik ihrer Struktur zu verstehen ist; zweitens mittels der „,funktionalen“ Dimension in einem übergeordneten politischen oder ökonomischen System, das über den regionalen und nationalen Kontext hinausreicht. Um die Bedeutung dieser beiden metropolitanen Dimensionen für die relative nationale ökonomische Performance der 40 Städte abzuschätzen, werden hier zwei Indikatoren herangezogen: erstens die Einwohnerzahl, zweitens der Status der Stadt im Netzwerk der Global Cities, gemessen in der GaWCConnectivity (vgl. DerudDer et al. 2010; TAYLOR et al. 2011).

Eine Korrelationsanalyse zeigt den Zusammenhang zwischen diesen beiden Dimensionen der Metropolisierung und verschiedenen Indikatoren der relativen nationalen ökonomischen Performance - dem Wirtschaftswachstum, dem relativen Wirtschaftswachstum im nationalen Kontext sowie dem Index des nationalen BIP/ Kopf (vgl. Tab. 4). Das Wirtschaftswachstum zeigt keinen Zusammenhang mit dem Global-City-Status und der Bevölkerungszahl - dies gilt sowohl für die Periode 2000 bis 2007 als auch für die Krisenjahre 2007 bis 2010. Ein Ergebnis, das der These, dass

\footnotetext{
8) Varianztest für die durchschnittliche jährliche Wachstumsrate 2000 bis 2007 sowie 2007 bis 2008 mit 0,000 signifikant.
} 


\begin{tabular}{|l|c|c|c|c|}
\cline { 2 - 5 } \multicolumn{1}{c|}{} & \multicolumn{2}{c|}{$\begin{array}{c}\text { Bevölkerungszahl } \\
\text { (N 40) }\end{array}$} & \multicolumn{2}{c|}{$\begin{array}{c}\text { Global-City-Status } \\
\text { (N 33) }\end{array}$} \\
\cline { 2 - 5 } & $\mathbf{2 0 0 0}$ bis 2007 & $\mathbf{2 0 0 7}$ bis 2010 & $\mathbf{2 0 0 0}$ bis 2007 & $\mathbf{2 0 0 7}$ bis 2010 \\
\hline $\begin{array}{l}\text { Durchschnittl. jährl. } \\
\text { BIP-Wachstum }\end{array}$ & $-0,083$ & 0,231 & 0,117 & $-0,021$ \\
\hline $\begin{array}{l}\text { Relatives durchschnittl. } \\
\text { jährl. BIP-Wachstum }\end{array}$ & 0,069 & $\mathbf{0 , 3 1 4}$ & 0,123 & $\mathbf{0 , 3 6 0 *}$ \\
\hline $\begin{array}{l}\text { Index des nationalen } \\
\text { BIP/Kopf }\end{array}$ & 0,244 & 0,302 & $\mathbf{0 , 6 7 1} * *$ & $\mathbf{0 , 7 1 0 * *}$ \\
\hline
\end{tabular}

Quelle: Eurostat Database 2013, GaWC-Database

Tab. 4: Korrelationsmaße zwischen metropolitanen Dimensionen (Global-City-Status, Einwohnerzahl) und ökonomischer Performance

große Metropolen oder Global Cities Wachstumsmaschinen der nationalen Volkswirtschaften darstellen, widerspricht und durch andere empirische Studien auch bestätigt wird (vgl. GorNig 2004; ESPON 2010, S. 45).

Diese Einschätzung wird allerdings etwas relativiert, wenn man das Wirtschaftswachstum der Städte um die nationalen Werte normiert. Das relative Wirtschaftswachstum zeigt nämlich einen schwachen Zusammenhang für beide metropolitanen Dimensionen, allerdings nur für die Periode ab 2007. Dies bedeutet also, eine große Bevölkerungszahl sowie ein hoher Global-City-Status scheinen mit einer gewissen Krisenresistenz einherzugehen. Ob der Begriff „Wachstumsmaschine“ angebracht ist, ist wohl fraglich. Auf jeden Fall handelt es sich dabei um jene Regionen, die im nationalen Kontext ein überdurchschnittliches Wirtschaftswachstum aufweisen. Dieser Befund deckt sich auch mit den vorhin (vgl. Kap. 5.1) festgestellten zunehmenden Divergenzen in den meisten Staaten.

Da es sich dabei tendenziell um die Hauptstädte der Staaten handelt, dürfte der vorhin angesprochene „Hauptstadtbonus“ auch hier einen Erklärungsfaktor darstellen - in allen zwölf Staaten sind die Hauptstädte die der Einwohnerzahl nach größten Städte sowie die bedeutendsten Global Cities (Ausnahme Berlin). Die zunehmenden nationalen Divergenzen in der Krise spiegeln sich ebenfalls in diesem (schwachen) Zusammenhang wider. Das ökonomische Entwicklungsniveau (Index des nationalen BIP/Kopf) innerhalb der Staaten steht zwar nicht mit der Bevölkerungsgröße, allerdings mit dem Status einer Stadt als Global City in einem deutlichen Zusammenhang, wobei dieser durch die Wirtschaftskrise noch verstärkt wird. Diese Analysen lassen jedoch die Richtung des Zusammenhangs offen; es bleibt unklar, ob die Dynamik der urbanen Ökonomien die Ursache oder das Ergebnis der nationalen Entwicklung ist. Aufgrund der Bedeutung der Metropolen für die jeweilige Gesamtwirtschaft wäre es durchaus auch denkbar, dass die volkswirtschaftliche Performance von jener der Metropolen abhängt und nicht umgekehrt. 


\section{Fazit}

Die empirische Analyse hat gezeigt, dass die Entwicklung der regionalen Disparitäten sowie der räumlichen Konvergenz/Divergenz innerhalb der Staaten der Eurozone eng an die makro-ökonomischen Ungleichgewichte gekoppelt ist. Die Prosperität von Regionen findet in einem relativ engen „Korridor“ statt, der durch die Entwicklung der Volkswirtschaften vorgegeben wird. Während in den Jahren vor der Wirtschaftskrise ein hohes Wirtschaftswachstum in den Ländern zu einer Abnahme regionaler Disparitäten geführt hat, gilt dies seit 2007 nicht mehr: Eine schrumpfende Ökonomie führt tendenziell zu ausgeprägteren Disparitäten. Hier handelt es sich, wie gesagt, um eine Tendenz und nicht um einen klaren Zusammenhang, denn in dem von der Krise hart getroffenen Portugal beispielsweise ist es zu einer leichten Konvergenz der räumlichen Entwicklung gekommen. Es zeigt sich, dass die räumliche Entwicklung in den Staaten und in deren Regionen von komplexen und teilweise widersprüchlichen Trends geprägt ist.

Diese Komplexität lässt sich teilweise erklären, wenn neben der Streuung auch die Entwicklung peripherer Regionen im jeweiligen nationalen Kontext berücksichtigt wird. Für die nationalen Peripherien der zwölf Staaten wurde es infolge der Wirtschaftskrise in Summe schwieriger, zum nationalen Durchschnitt aufzuschließen. Je höher also das ökonomische Entwicklungsniveau von Regionen innerhalb eines Staates ist, umso stärker war - tendenziell - ihr relatives Wirtschaftswachstum. Umgekehrt zeigte sich für Portugal, dass die konvergente Entwicklung eher durch die Krise der Zentren und weniger durch die Prosperität der Peripherie ausgelöst wurde. Die spezifischen nationalen Kontexte machen eine allgemeine und zugleich eindeutige Aussage zur Entwicklung peripherer Regionen schwierig, wenngleich seit Ausbruch der Krise der Trend zu ausgeprägteren regionalen Divergenzen hin zu gehen scheint.

Die wirtschaftliche Entwicklung der Metropolen zeigt, wie eng diese an die jeweiligen Volkswirtschaften gekoppelt ist. Nahezu alle der 40 hier untersuchten Metropolen bewegen sich - insbesondere seit 2007 - in einem sehr schmalen „Korridor“ von $\pm 0,5 \%$ um das nationale Wirtschaftswachstum. Der Eindruck einer starken Koppelung an die nationale Wirtschaftsentwicklung bestätigt damit ältere Studien (vgl. ESPON 2010), wobei mit der vorliegenden Untersuchung nicht geklärt werden kann, ob die Volkswirtschaften die Performance der Metropolen beeinflussen oder ob es möglicherweise eine zirkuläre Kausalität gibt.

Dieser Beitrag hat versucht die Notwendigkeit aufzuzeigen, regionale Entwicklungen im volkswirtschaftlichen Kontext zu sehen sowie die aktuelle Wirtschaftskrise mit ihren massiven makro-ökonomischen Verwerfungen in den Fokus raumwissenschaftlicher Debatten zu stellen. Sieht man räumliche Entwicklungen, die Dynamik urbaner Zentren sowie peripherer Regionen, lediglich durch die Brille des „Europas der Regionen“, werden wichtige und raumrelevante Prozesse und Strukturen ausgeblendet. Die Divergenzen in Folge der aktuellen Wirtschaftskrise sind nur ein Anknüpfung spunkt, der das komplexe Verhältnis zwischen Region, Staat und supranationaler (europäischer) Ebene verdeutlicht. Andere nationalstaatliche Faktoren wie die räumliche Staatsorganisation (föderale oder zentralistische Staaten) oder die spezifischen nationalen 
makro-ökonomischen Arrangements (vgl. „Varieties of Capitalism“) wären in diesem Kontext zu berücksichtigen. Auch anhand dieser Faktoren könnte gezeigt werden, dass das Europa der Regionen - trotz Globalisierung und europäischer Integration - nach wie vor in ein Europa der Staaten eingebettet ist.

\section{Literaturverzeichnis}

Amable B. (2003), The diversity of modern capitalism. Oxford, Oxford University Press.

ANGEW J. (1994), The territorial trap: the geographical assumptions of international relations theory. In: Review of International Political Economy, 1, 1, S. 53-80.

Aswestopoulos W. (2013), Reparationen, Rückzahlungen von Krediten oder Revanche? Telepolis, Heise Verlag. 25.4.2013. - http://www.heise.de/tp/artikel/38/38997/1.html

Barro R., Sala-I-Martin X. (1991), Convergence across States and Regions. In: Brookings Papers on Economic Activity, 1, S. 107-182.

BeCKer W. (2008), Der Euro wird zehn. Frankfurt am Main, Deutsche Bank.

Blotevogel H.H. (2010), Raumordnung und Metropolregionen. In: Geographische Rundschau, 11, S. 14-21.

BoldRIN M., CANOva F. (2001), Inequality and convergence in Europe's regions: reconsidering European regional policies. In: Economic Policy, 4, S. 3-49.

BRENNER N. (2004), New state spaces. Urban Governance and the rescaling of statehood. Oxford, Oxford University Press.

Breuss F. (2011), Makro-ökonomische Ungleichgewichte. In: Wirtschaftspolitische Blätter, 58, 3, S. 409-428.

Canova F. (2004), Testing for convergence clubs in income per capita: A predicitve density approach. In: International Economic Review, 45, 1, S. 49-77.

Castells M. (1998), The information age. The rise of the network society. Malden, Mass., Blackwell.

Derudder B., Taylor P., Ni P., De Vos A., Hoyler M., Hanssens H., Bassens D., Huang J., Witlox F., Shen W., YAng X. (2010), Pathways of Change: Shifting Connectivity in the World City Network 2000-2008. In: Urban Studies, 47, 9, S. 1861-1877.

Dijkstra L. (2009), Metropolitan Regions in the EU. Metropolitan Regions in the EU. Brüssel, European Union.

Dijkstra L., Garcilazo E., McCann P. (2013), The Economic Performance of European Cities and City Regions: Myths and Realities. In: European Planning Studies, 21, 3, S. 334-354.

DÜHR S. (2013), Raumordnerische und planungspolitische Regionalisierungen. In: GeBHART H., Glaser R., Lentz S. (Hrsg.), Europa, eine Geograhie, S. 212-221. Berlin, Springer.

Dunford M. (2005), Old Europe, New Europe and the USA. Comparative economic Performance, Inequality and market-led Models of Development. In: European Urban and Regional Studies, 12, 2, S. 149-176.

EsPing-Andersen G. (1990), The three worlds of welfare capitalism. Cambridge, Polity.

ESPON (Hrsg.) (2004), Espon 1.1.1. Potentials for polycentric development in Europe. Project report. Luxemburg, Espon.

ESPON (Hrsg.) (2010), FOCI Future Orientations for Cities. Final Report 2010. Luxemburg, ESPON.

Europäische Kommission (Hrsg.) (1988), Europe 1992. The Overall Challenge. Cecchini-Report. Bruxelles/Brussel, Europäische Kommission. 
Europäısche Kommission (Hrsg.) (1999), Europäisches Raumentwicklungskonzept. Luxemburg, Europäische Kommission.

Europäische Kommission (Hrsg.) (2013), Report from the Commission of the European Parliament and the Council. Brüssel, Europäische Kommission.

Eurostat Database (2013) - http://epp.eurostat.ed.europa.eu/portal/page/portal/waste/data/ database (letzter Zugriff November 2013)

Fischer K., Reiner C., Staritz C. (Hrsg.) (2010), Globale Güterketten. Weltweite Arbeitsteilung und ungleiche Entwicklung (= Historische Sozialkunde, 29). Wien, Promedia/Südwind.

Fromhold-Eisebith M. (1995), Das „kreative Milieu“ als Motor regionalwirtschaftlicher Entwicklung. Forschungstrends und Erfassungsmöglichkeiten. In: Geographische Zeitschrift, 83,1, S. 30-47.

Geppert K., Happich M., Stephan A. (2005), Regional Disparities in the European Union: Convergence and Agglomeration. Berlin, German Institute for Economic Research.

Giese E., Mossig I., Schröder H. (2011), Globalisierung der Wirtschaft. Eine wirtschaftsgeographische Einführung. Paderborn, Schöningh.

GorNIG M. (2004), Deindustrialisierung und Globalisierung: Folgen für die ökonomische Bedeutung (europäischer) Städte. In: Siebel W. (Hrsg.), Die europäische Stadt, S. 385-395. Frankfurt am Main, Suhrkamp.

Grote M.H. (2003), Die Evolution des Finanzplatzes Frankfurt. In: Geographische Zeitschrift, 91, 3-4, S. 200-217.

Hadimichalis C. (2011), Uneven geographical development and socio-spatial justice and solidarity: European regions after the 2009 financial crisis. In: European Urban and Regional Studies, 18, 3, S. 21.

Hall P.A., Soskice D. (Hrsg.) (2001), Varieties of Capitalism. The Institutional Foundations of Comparative Advantage. Oxford, Oxford University Press.

Hamnett C. (2003), Unequal City. London in the Global Arena. London, Routledge.

Hancké B. (Hrsg.) (2009), Debating Varieties of Capitalism. A Reader. Oxford, Oxford University Press.

Hasse S. (2010), Spanien: Das nächste Sorgenkind Europas. Die Auswirkungen der Immobilienpreisblase. In: Geographische Rundschau, 11, S. 60-65.

Häussermann H., Haila A. (2005), The European City: A Conceptual Framework and Normative Project. In: Kazepov Y. (Hrsg.), Cities of Europe. Changing Contexts, Local Arrangements, and the Challenge to Urban Cohesion, S. 43-64. Oxford, Blackwell.

Heeg S. (1996), Endogene Potentiale oder footloose captitalism? Einige Anmerkungen zur sozialen Regulation des Raums. In: BRUCH M., KREBS H.-P. (Hrsg.), Unternehmen Globus: Facetten nachfordistischer Regulation, S. 199-223. Münster, Westfälisches Dampfboot.

HeEg S., Dörry S. (2009), Leerstände und Bauboom - Büroimmobilien nur noch ein Anlageprodukt? In: Forschung Frankfurt, 3, S. 30-36.

Heintel M. (1998), Einmal Peripherie - immer Peripherie? Szenarien regionaler Entwicklung anhand ausgewählter Fallbeispiele. Wien, Institut für Geographie, Universität Wien.

Hudson R. (2003), European Integration and new Forms of Uneven Development. In: European Urban and Regional Studies, 10, 1, S. 49-67.

Hudson R., Williams A.M. (Hrsg.) (1999), Divided Europe. London, Sage.

Husa K., Wohlschlägl H. (1999), Vom „Emerging Market“ zum „Emergency Market“: Thailands Wirtschaftsentwicklung und die „Asienkrise“. In: Parnreiter C., Novy A., Fischer K. (Hrsg.), Globalisierung und Peripherie. Umstrukturierung in Lateinamerika, Afrika und Asien, S. 209-236. Wien, Brandes \& Aspel/Südwind.

International Monetary Fund IMF (Hrsg.) (2011), World Economic Outlook: Rebalancing Growth. Washington, International Monetary Fund. 
JESSOP B. (1997), Die Zukunft des Nationalstaats - Erosion oder Reorganisation? Grundsätzliche Überlegungen zu Westeuropa. In: Becker St., S Ablowski T., Schumm W. (Hrsg.), Jenseits der Nationalökonomie? Weltwirtschaft und Nationalstaat zwischen Globalisierung und Regionalisierung, S. 50-96. Hamburg, Argument.

Karanikolos M., Mladovsky P., Cylus J., Thomson S., Basu S., Stuckler D., Mackenbach J.P., McKeE M. (2013), Financial crisis, austerity, and health in Europe. In: The Lancet. Healt in Europe, 7, S. 1-9.

Kaufmann F.-X. (2003), Der deutsche Sozialstaat im internationalen Vergleich. Frankfurt am Main, Suhrkamp.

Kazepov Y. (2005), Cities in Europe: Changing Contexts, Local Arrangements, and the Challenge to Social Cohesion. In: KAzepov Y. (Hrsg.), Cities of Europe, S. 3-42. Oxford, Blackwell.

Keating M. (1997), The Political Economy of Regionalism. In: Keating M., Loughlin J. (Hrsg.), The Political Economy of Regionalism. London, Frank Cass.

Keil R., Brenner N. (2003), Globalisierung, Stadt und Politik. In: Scharenberg A., Schmidtke O. (Hrsg.), Das Ende der Politik? Globalisierung und der Strukturwandel des Politischen, S. 254-276. Münster, Westfälisches Dampfboot.

Kemper F.-J. (2006), Metropolen: Merkmale, Konzepte, Perspektiven. In: Schultz H.-D. (Hrsg.), Metropolitanes \& Mediterranes. Beiträge aus der Humangeographie (= Berliner Geographische Arbeiten, 107), S. 1-20. Berlin, Geographisches Institut, Humboldt-Universität Berlin.

KNIELING J. (Hrsg.) (2009), Metropolregionen. Innovation, Wettbewerb, Handlungsfähigkeit. Metropolregionen und Raumentwicklung, Band 3. Hannover, ARL.

KNIELING J. (2009), Metropolregionen: Zukunftsthema oder Zeitgeist? In: KNIELING J. (Hrsg.), Metropolregionen. Innovation, Wettbewerb, Handlungsfähigkeit, S. 1-8. Hannover, ARL.

Krugman P. (1991), Geography and Trade. Leuven, Cambridge/Mass., Leuven University Press.

Kujath H.J. (2009), Leistungsfähigkeit der Metropolregionen in der Wissensökonomie - Die institutionentheoretische Sicht. In: KNIELING J. (Hrsg.), Metropolregionen. Innovation, Wettbewerb, Handlungsfähigkeit, S. 200-222. Hannover, ARL.

Kunzmann K.R. (1992), Zur Entwicklung der Stadtsysteme in Europa. In: Mitteilungen der Österreichischen Geographischen Gesellschaft, 134, S. 25-50.

Kunzmann K.R., Wegener M. (1991), The Pattern of Urbanisation in Western Europe (= Berichte aus dem Institut für Raumplanung, 28). Dortmund, Institut für Raumplanung.

Lessmann C. (2005), Regionale Disparitäten in Deutschland und ausgesuchten OECD-Staaten im Vergleich. In: ifo Dresden berichtet, 12, 3, S. 25-33.

LESSMANN C. (2009), Fiscal decentralization and regional disparity: evidence from cross-section and panel data. In: Environment and Planning A, 41, S. 2455-2473.

Leyshon A., Thrift N. (1997), Money/Space: Geographies of Monetary Transformation. London - New York, Routledge.

Lijphart A. (1999), Patterns of Democracy. Government Forms and Performance in Thirty-Six Countries. New Haven, Yale University Press.

LÜthi S., Thierstein A., Bentlage M. (2010), Standortverflechtungen in der Wissensökonomie. In: Geographische Rundschau, 11, S. 46-53.

Ma X., Timberlake M. (2012), World City Typologies and National System Deterritorialisation: USA, China and Japan. In: Urban Studies, Online, S. 1-21.

McNeill D. (2006), Performing European Space? In: European Urban and Regional Studies, 13,1, S. 83-87.

Melo P.C., Graham D.J., Noland R. (2009), A meta-analysis of estimates of urban agglomeration economies. In: Regional Science and Urban Economics, 39, 3, S. 332-342. 
Musgrave R., Musgrave P., Kullmer L. (1992), Die öffentlichen Finanzen in Theorie und Praxis. 3. Band. Tübingen, Mohr.

Musil R. (2012), Konvergenz durch Dezentralisierung? Die Entwicklung der europäischen Peripherien vor dem Hintergrund der räumlichen Staatsorganisation. In: Schmied D. (Hrsg.), Peripherien in Europa zwischen Rückbau und Umbau, S. 1-36. Göttingen, Cuvillier.

Österreichische RaumordnungsKonferenz (ÖROK) (Hrsg.) (2009), EU-Kohäsionspolitik in Österreich 1995-2007. Eine Bilanz (= ÖROK Schriftenreihe, 180). Wien, ÖROK.

PAAsi A. (2001), Europe as a Social Process and Discourse. Considerations of Place, Boundaries and Identity. In: European Urban and Regional Studies, 8, 1, S. 7-28.

Palme G., Musil R. (2012), Wirtschaftsgeographie. Braunschweig, Westermann.

PeCK J. (2000), Discussant‘s commentary. Annual Conference, Institute of British Geographers, Brighton, Panel on Economics and Politics of Scale.

Petrakos G., Kallioras D., Anagnostou A. (2011), Regional convergence and growth in Europe: unterstanding patterns and determinants. In: European Urban and Regional Studies, 18, 4, S. 375-391.

Porter M. (2000), Locations, clusters, and company strategy. In: Clark G., Al E. (Hrsg.), The Oxford Handbook of Economic Geography, S. 253-274. Oxford, Oxford University Press.

Porter M.E. (1999), Wettbewerb und Strategie. München, Econ.

Qian Y., Weingast B. (1997), Federalism as a commitment to preserving market incentives. In: Journal of Economic Perspectives, 11, S. 83-91.

QuAH D. (1996), Regional convergence clusters across Europe. In: European Economic Review, 40, S. 951-958.

Robertson R. (1995), Glocalization: time-space and homogeneity-heterogeneity. In: LASH M., Robertson R. (Hrsg.), Global Modernities, S. 25-44. London, Sage.

Rodriguez-Pose A. (1999), Convergence or divergence? Types of regional responses to socioeconomic change in Europe. In: Tijdschrift voor Economische en Sociale Geografie, 90, 4, S. 363-378.

Romer P. (1986), Increasing Returns and Long-run Growth. In: Journal of Political Economy, 94, S. 1002-1037.

SALA-I-Martin X. (1996), Regional cohesion: Evidence and theories of regional growth and convergence. In: European Economic Review, 40, S. 1325-1352.

SASSEN S. (1991), The global city. London - New York - Tokyo - Princeton, Princeton University Press.

SASSEN S. (2006), Territory, Authority, Rights. From medieval to global assemblages. Princeton - Oxford, Princeton University Press.

Schamp E.W. (2011), Europas jüngstes Finanzzentrum. Der Aufstieg Frankfurts zur europäischen Finanz-Metropole. In: Matznetter W., Musil R. (Hrsg.), Europa: Metropolen im Wandel, S. 53-66. Wien, Mandelbaum.

SChätZl L. (2011), Wirtschaftsgeographie 1. Theorie. Paderborn, Schöningh.

Schratzenstaller M. (2005), Neuer Finanzausgleich und Stabilitätspakt - keine grundlegenden Änderungen. In: WIFO-Monatsberichte, 1, S. 49-60.

Shankar R., Shah A. (2003), Bridging the economic divide within countries - a scorecard on the performance of regional policies in reducing regional income disparities. In: World Development, 31, S. 20.

SinN H.W. (2012), Die Target-Falle. München, Hanser.

SLOTERDiJK P. (2008), Theorie der Nachkriegszeiten. Frankfurt am Main, Suhrkamp.

TAYLOR P. (2004), World City Network. A global urban analysis. London - New York, Routledge.

Taylor P.J., Ni P., Derudder B., Hoyler M., Huang J., Witlox F. (Hrsg.) (2011), Global Urban Analysis. A Survey of Cities in Globalization. London - Washington, Earthscan. 
Vorauer K. (1997), Europäische Regionalpolitik - Regionale Disparitäten. Theoretische Fundierung, empirische Befunde und politische Entwürfe (= Münchner Geographische Hefte, 77). Passau, LIS-Verlag.

Vorauer-Mischer K . (2009), Europäische Regionalpolitik - vom Ausgleichs- zum Wachstumsziel? In: Musil R., Staudacher C. (Hrsg.), Mensch. Raum. Umwelt. Entwicklungen und Perspektiven der Geographie in Österreich, S. 184. Wien, Österreichische Geographische Gesellschaft.

Wolf M. (2009), Die Organisierung des sozialen Krieges: zur staatspolitischen Dimension der Hartz-IV-Reform. Grundrisse, Online-Manuskript. - http://www.grundrisse.net/ grundrisse31/organisierung_sozialen_krieges.htm

ZeHner K. (2011), Von der Hauptstadt des Empire zur Alpha Global City. Die Stadtentwicklung Londons im Zeichen von Globalisierung und Stadtentwicklungspolitik. In: MATZNETTER W., Musil R. (Hrsg.), Europa: Metropolen im Wandel, S. 39-51. Wien, Mandelbaum.

Zeller C. (2003), Bausteine zu einer Geographie des Kapitalismus. In: Zeitschrift für Wirtschaftsgeographie, 47, 2-3, S. 16. 